Mercè Prats (Université de Reims Champagne-Ardenne)

\title{
LE PHÉNOMÈNE HUMAIN DE PIERRE TEILHARD DE CHARDIN, UN MÉMOIRE SCIENTIFIQUE?
}

\begin{abstract}
Jesuit and palaeontologist Pierre Teilhard de Chardin died suddenly on the $4^{\text {th }}$ of April, 1955, leaving a large part of his work unpublished. Throughout the rest of the year, the press announced the imminent publication of The Phenomenon of Man, presented as the scientific side of his work. By fall, the book was published, accompanied by an impressive list of prestigious signatures. But over time, this tacit consensus loses its vigour and the question of the book's scientific nature makes a strong comeback. The first interrogations arise in the early 1960s. These interrogations sometimes concern his scientific work, sometimes his philosophical-theological work, but almost never the man himself. To follow the reception of The Phenomenon of Man comes down to following the line drawn by Teilhardism. This ism is associated with Teilhard de Chardin's thought, which takes its shape at the heart of the Glorious Thirty in France, before setting alight many other countries, and which seems to decline after crossing the threshold of 1965.
\end{abstract}

Keywords: Teilhard, Teilhardisme, Phenomenon of Man, scientific and philosophical-theological work.

\section{Introduction}

Dès que, le vendredi 21 octobre, j'ai manié le premier exemplaire du Phénomène Humain sorti des presses du Seuil, une joie mêlée de tristesse m'a étreint le cœur, joie devant cette réparation posthume que représente, pour le Père Teilhard de Chardin, ce volume à la sobre couverture gris-bleu, tristesse en

\footnotetext{
- Adresse pour correspondance: 13 le Montferré, 51500 Trois Puits, France. Email: m.merce@wanadoo.fr.
} 
pensant au bonheur que nous aurions eu, au cours d'une fête intime, à lui offrir cet in-80 carré, résultat de plus de deux ans de travail - 1938-1940 - qu'il avait lui-même complété en 1948 et que ses amis théologiens avaient lu et relu avec admiration. ${ }^{1}$

Ces mots sont extraits de la première note de lecture publiée dans la presse à peine quelques jours après la publication du Phénomène humain. L'ouvrage n'est pourtant pas d'une lecture facile mais Claude Cuénot, l'auteur de cette note, n'avait pas besoin de le lire, ayant eu ce texte dans les mains maintes fois auparavant. Il avait suivi de près la carrière de Teilhard de Chardin, un ami de son père, le célèbre biologiste Lucien Cuénot ${ }^{2}$. La publication de ce livre éveille en lui un sentiment partagé. À la joie de voir enfin l'œuvre inédite mise à disposition du grand public se mêle la tristesse de savoir que Teilhard de Chardin ne l'aura jamais vue imprimée. Cette interdiction de publication du vivant de l'auteur avait une origine lointaine.

En 1922, Teilhard rédige une Note sur quelques représentations historiques possibles du Péché originel ${ }^{3}$. Ce document parvient à Rome. Accusé d'avoir une vision peu orthodoxe du péché originel, Teilhard se voit privé de sa chaire de géologie à l'Institut Catholique de Paris puis renvoyé en Chine, poursuivre ses travaux sur le terrain. Ses supérieurs craignent une réaction du Saint-Office, l'autorité chargée de surveiller les publications, devant une pensée qui paraît fort audacieuse. Cet incident, somme toute mineur, l'entoure à jamais de suspicion et conditionne toute sa carrière. Il était loin d'être le seul clerc dans cette situation. Cette ambiance empreinte de soupçon, héritée de la crise moderniste du début du siècle, frappe bon nombre de religieux. Devant l'impossibilité de diffuser leur œuvre sans encourir de sanction, ils développent parfois des techniques de dissimulation. Par exemple, Alfred Loisy usait alternativement du pseudonyme Isidore Desprès ou encore de A. Firmin ${ }^{4}$. L'abbé Turmel était connu pour avoir utilisé plus d'une quinzaine de noms d'emprunt $^{5}$. De son côté, Teilhard inaugure un mode de contournement particulier: la diffusion de l'œuvre sous la forme polycopiée. La diffusion est limitée, certes, mais elle a le mérite de ne pas être nulle. Il en découle de ce mode de circulation un bénéfice supplémentaire: si l'œuvre n'a pas d'existence officielle, elle ne pourra pas être ajoutée à l'Index, la liste des livres que les fidèles ne doivent pas lire car, s'ils se trouvent sur cette liste c'est que le Saint-Office les considère dangereux, capables de troubler la foi ou les mœurs ${ }^{6}$.

${ }^{1}$ C. Cuénot, Pierre Teilhard de Chardin: Le Phénomène humain in FTdC.

${ }^{2}$ Lucien Cuénot (1866-1951) est un biologiste de grande renommée, membre de l'Académie des sciences, toujours demeuré attaché à sa ville, Nancy. Son fils, Claude Cuénot, est plutôt attiré par des études littéraires. Sa thèse de doctorat porte sur Verlaine. Claude Cuénot profite parfois de l'importance acquise par son père dans le milieu des scientifiques mais il en est tout à la fois éclipsé. Dans ces pages, il sera souvent question de Claude Cuénot étant donnée l'importance qu'il acquiert dans la publication posthume de l'œuvre de Teilhard de Chardin.

${ }^{3} \mathrm{Cf}$. P. Teilhard de Chardin, Note sur quelques représentations historiques possibles du péché originel.

${ }^{4}$ Cf. P. Colin, L'Audace et le Soupçon, p. 139.

${ }^{5}$ Cf. É. Poulat, Histoire, Dogme et Critique dans la crise moderniste.

${ }^{6}$ Cf. J.-B. Amadieu, La Littérature française au XIX siècle mise à l'Index, pp. 13-74. 
La condamnation de l'œuvre est à craindre mais pas seulement. Le SaintOffice peut procéder à l'excommunication du prêtre. Loisy a été excommunié en 1908. L'abbé Turmel, démasqué tardivement, est excommunié en 1930. Le cas de Teilhard est exceptionnel. S'il se meut dans les marges, il semble échapper aux sanctions. En effet, l'homme a un charisme particulier que ses supérieurs n'ignorent pas. En 1948, il accomplit son seul et unique voyage à Rome. Le Supérieur Général de son ordre semble lui laisser espérer une possible publication du Phénomène humain ainsi qu'une éventuelle reprise d'une chaire au Collège de France, à la suite de l'abbé Breuil. Une fois arrivé à Rome, le jésuite comprend les vraies raisons qui ont conduit le $P$. Janssens à le faire venir. Il l'écrit ainsi dans une lettre à René d'Ouince, son supérieur à Paris: Je commence à comprendre la tactique: on me sort et on me montre, comme un animal inoffensif. C'est amusant; et au fond c'est si gentil. ${ }^{1}$ Il fallait qu'il soit $\mathrm{vu}$, que les membres du Saint-Office le rencontrent pour qu'ils soient convaincus de sa bonne foi. Ce voyage à Rome se solde par un nouveau refus de publication mais, indéniablement, cette visite a joué en sa faveur. Aucune sanction ne sera jamais prise à son égard, pas d'excommunication, pas de mise à l'Index. Il poursuit sa carrière de paléontologue, notamment en Chine, ce qui ne fait qu'accroître sa légitimité scientifique. Il est fort possible que, à Rome, les théologiens aient vu chez lui une sorte d'apologétique vivante ${ }^{2}$ car ce paléontologue avait fait une brillante carrière scientifique sans jamais rien renier de son état de jésuite. Dès 1912, il avait été l'élève du professeur Marcelin Boule au Muséum national d'histoire naturelle. Le professeur Boule avait acquis une grande renommée avec la description de l'homme néandertalien découvert à la Chapelle-aux-Saints. Après une thèse, en 1922, sur les Mammifères de l'Éocène inférieur français et leurs gisements, Teilhard avait poursuivi ensuite ses recherches en Chine et participe à la découverte du Sinanthrope. En 1931, il est invité à faire partie de l'équipe qui se lance dans l'aventure de la Croisière Jaune. Il accomplit de nombreux voyages d'études en Éthiopie, Inde, Birmanie, Afrique du Sud, avant de terminer sa carrière aux États-Unis, soutenu par la Wenner-Gren Foundation.

Si l'Église ne lui a jamais refusé la publication de ses écrits scientifiques, ses écrits philosophico-théologiques n'ont jamais été publiés de son vivant. Cette note écrite par Teilhard en 1922 avait déclenché la suspicion car elle portait sur le péché originel, une question qui inquiète tout particulièrement l'Église. Sans condamner les théories de l'évolution, l'Église refuse l'hypothèse du polygénisme. Cette position sera précisée par Pie XII, en 1950, avec l'encyclique Humani generis. Le polygénisme est incompatible avec la doctrine du péché originel, celle d'un premier couple qui serait à l'origine du péché, celui qui explique l'existence du mal ${ }^{3}$. Ce n'est pas une question périphérique. Si le Christ est venu sur Terre c'est bien pour effacer le péché commis par ce couple premier. S'il n'y a pas de péché originel c'est toute la doctrine qui s'effondre.

\footnotetext{
${ }^{1}$ Lettre de P. Teilhard de Chardin à R. d'Ouince, 7 octobre 1948 in: FTdC.

${ }^{2}$ R. d'Ouince, Un prophète en procès: Teilhard de Chardin, p. 121.

${ }^{3}$ Cf. F. Laplanche, La Crise de l'origine, p. 344.
} 
La pensée de Teilhard de Chardin a inquiété les autorités romaines. La publication de ses œuvres n'a jamais été autorisée.

Cependant, Teilhard lutte pour parvenir à la diffusion de l'œuvre jusqu'à son dernier souffle. Dans ce combat, il n'est pas seul. Deux cercles suivent de près ses travaux. D'une part, quelques amis voudraient faire connaître ces écrits au plus vite car, à leurs yeux, ils regorgent de possibilités à produire des conversions, des retours à la foi. Dans leur esprit, Rome viendra reconnaître un jour les mérites de ce pionnier de la foi. Le nombre de lecteurs ne cesse d'augmenter depuis que Teilhard a mis en circulation ses papiers. Ils sont copiés de bonne heure par ses amis avant qu'il ne se mette en place une sorte de petite industrie de ronéotypes, relayée par Jeanne Mortier en $1939^{1}$. D'autre part, quelques clercs voudraient le pousser à utiliser des termes plus orthodoxes, à les préciser, à amender certains écrits de manière à rendre l'œuvre publiable, tel est le mot de $\mathrm{M}^{\text {gr }}$ de Solages ${ }^{2}$. Avec le P. de Lubac, ces deux clercs redoublent d'efforts pour que Teilhard précise sa pensée ${ }^{3}$. En 1947, avant de présenter une nouvelle fois le Phénomène humain à Rome, ils organisent une rencontre sur les terres de Solages, à Carmaux. Teilhard repart de ce Concile de Carmaux avec de nombreuses corrections à entreprendre. Il opère quelques modifications et procède à quelques ajouts sur le texte du Phénomène humain dont un d'importance majeure: l'avertissement, dont voici un extrait:

Pour être correctement compris, le livre que je présente ici demande à être lu, non pas comme un ouvrage métaphysique, encore moins comme une sorte d'essai théologique, mais uniquement et exclusivement comme un mémoire scientifique. Le choix même du titre l'indique. Rien que le Phénomène. Mais aussi tout le Phénomène. ${ }^{4}$

Ce livre est une tentative d'expliquer la place de l'homme dans la nature. Il $s$ 'insère aussi dans une vieille querelle entre science et foi. Dans une immense fresque, Teilhard expose la spiritualisation de la matière suivant des paliers qui vont de l'atome à la molécule, puis à la cellule pour arriver à l'homme. Ce présupposé comprend des implications philosophiques. Pourtant, dans l'avertissement que nous présentons ci-dessus, Teilhard insiste: l'œuvre doit être lue comme un mémoire scientifique. Pour le moment, le devenir de ce texte reste

\footnotetext{
${ }^{1}$ Jeanne Mortier (1892-1982) menait une vie de femme consacrée. Elle avait entendu Teilhard en 1938, au cours d'une conférence, et avait voulu le rencontrer. Très rapidement, elle en est devenue son plus fidèle soutien parmi les laïcs. Teilhard, absent de Paris la plupart du temps, s'en remettait à Jeanne Mortier et lui confiait la distribution des textes polycopiés. Cette histoire de la circulation des polycopiés de Teilhard fait l'objet du préambule de notre thèse: M. Prats, Le Teilhardisme.

${ }^{2}$ Cf. Lettre de Br. de Solages à H. de Lubac, 24 avril 1955 in: CAECHL.

${ }^{3}$ Bruno de Solages (1895-1983) et Henri de Lubac (1896-1991) n’ont jamais cessé de soutenir Teilhard de Chardin, l'homme et l'œuvre. Bruno de Solages, recteur de l'Institut catholique depuis 1931, peut le défendre ouvertement tandis que Henri de Lubac, frappé par la crise de Fourvière, agit plutôt dans l'ombre jusqu'au moment où, au début des années 1960, il est invité à participer à la Commission théologique préparatoire du concile Vatican II. Cette réhabilitation représente un tournant important, pour lui et pour Teilhard de Chardin posthume. Bruno de Solages et Henri de Lubac se consultent toujours avant d'agir.

${ }^{4}$ P. Teilhard de Chardin, Le Phénomène Humain, p. 21.
} 
incertain. Après ce dernier refus romain, et alerté par une sévère crise cardiaque, Teilhard s'inquiète. De ces deux cercles qui l'entourent, l'un composé de clercs et l'autre porté par Jeanne Mortier, il choisit cette amie audacieuse comme légataire. En effet, quelques jours après le décès, les lecteurs apprennent par la presse l'existence d'un testament en faveur de sa secrétaire, son bras droit, Jeanne Mortier ${ }^{1}$. Dès les premiers jours, elle se dit prête à suivre la vocation de Teilhard et c'est ainsi qu'elle le fait entendre ${ }^{2}$. Impossible de lui ravir la légitimité du testament. Sa première mission sera celle de l'édition mais, ayant toute l'œuvre en main, pourquoi commence-t-elle par publier le Phénomène humain? Comment réussit-elle à échapper aux sanctions? Est-ce le soutien des scientifiques qui donne vie au teilhardisme?

Nous verrons, dans un premier temps, quels ont été les soutiens que les scientifiques apportent à l'édition de l'œuvre et à celle du Phénomène humain en particulier. Nous suivrons ensuite les premières contestations, timides, avant qu'elles ne deviennent de sérieuses remises en question, une dizaine d'années après les débuts de cette publication posthume. La voix des scientifiques et ses différentes expressions contribue à dessiner cette vague de teilhardisme qui déferle en France, avant de s'internationaliser, en ces années 1950-1960.

\section{Publier l'œuvre du jésuite-paléontologue}

Jeanne Mortier se fait connaître comme légataire des œuvres quelques jours après le décès de Teilhard ${ }^{3}$. Cependant, dans cette tâche, Teilhard avait prévu qu'elle ne soit pas seule. Le testament qu'il avait rédigé en sa faveur stipulait l'existence d'un petit comité - ce sont les mots utilisés par Teilhard dans le testament -, avec quelques noms qui viendraient prendre le relais en cas de défaillance de l'héritière ${ }^{4}$. Teilhard avait noté trois noms: Jean Piveteau, un de ses collègues paléontologues, André George, à la tête des éditions scientifiques chez Albin Michel, et François Richaud, un psychiatre avec lequel il avait échangé une correspondance. Néanmoins, quelques années après, Teilhard remplace Richaud par Claude Cuénot, ancien élève de l'École Normale supérieure et fils de son ami Lucien Cuénot. Claude Cuénot est le seul dans ce petit comité à ne pas faire œuvre scientifique mais sa force de caractère a été perçue comme un atout dans ce projet ${ }^{5}$. Ce petit comité se réunit pour la première fois le 23

\footnotetext{
1 Jeanne Mortier suivait, généralement, les instructions de Teilhard. Elle pouvait aussi avoir quelques initiatives. Par exemple, en 1951, Jeanne Mortier avait voulu offrir à Teilhard, pour ses 70 ans, un exemplaire imprimé du Cœeur de la Matière. Pour cela, elle se rend à Rome, l'ouvrage en main, et explique aux supérieurs jésuites comment cette œuvre, qui circule et produit des conversions, serait bien plus efficace dans une version éditée. Ce qui devait être un mode de diffusion discret était alors présenté comme une pratique courante. Teilhard a été rappelé à l'ordre. Peu après cet incident, loin de s'en offusquer, Teilhard confie la totalité de son œuvre à Jeanne Mortier (testament daté du 2 juillet 1951). Cf. M. Prats, Le Teilhardisme.

${ }^{2}$ Cf. Lettre de J. Mortier au P. Villain, le 12 mai 1955 in: ASJF, fonds Teilhard de Chardin 6-4.

${ }^{3}$ Coupures de presse adressées à P. Flamand (éditions du Seuil) par J. Mortier, 17 avril 1955 in: IMEC.

${ }^{4}$ Ce testament est rappelé dans toutes les biographies de Teilhard et cité in extenso in: H. de Lubac, $L a$ Pensée religieuse du P. Teilhard de Chardin, pp. I-XX.

${ }^{5}$ Cf. Lettre de P. Teilhard de Chardin à J. Mortier, 25 janvier 1955 in: P. Teilhard de Chardin, Lettres à Jeanne Mortier, p. 173.
} 
avril 1955, sous l'égide de Jeanne Mortier. Plusieurs décisions sont prises à cette occasion.

\subsection{Le Phénomène humain s'impose}

Ce comité assume les deux fonctions prévues par le testament: conservation et diffusion de l'œuvre. La conservation ne sera pas une mince affaire car l'œuvre est éparpillée, mal répertoriée, les différentes versions coexistent ... . Ce travail se fera progressivement; il n'interdit pas la diffusion immédiate de l'Euvre, ce que Jeanne Mortier voit comme une urgence absolue. Au cours de cette rencontre, l'ordre de publication est discuté, ses modalités aussi. La situation de la légataire, prise entre deux feux, est délicate. D'un côté les pères jésuites voudraient ralentir cette édition pendant que l'éditeur voudrait publier au plus vite. Au lendemain de cette réunion, Mortier reçoit une lettre de $\mathrm{M}^{\mathrm{gr}} \mathrm{de}$ Solages. Inquiet de la voir se lancer dans une publication hâtive, il se résout à la conseiller, faute de pouvoir l'arrêter:

Le moment n'est pas favorable pour publier. Néanmoins c'est bien par le Phénomène humain qu'il faut commencer. [...] Nous parlerons de tout cela, j'espère, de vive voix à Paris quand je viendrai vous rendre visite, sans doute en octobre prochain. Bien entendu, si je peux dès maintenant continuer à rendre service à la cause, comme il [Teilhard] disait, vous n'avez qu'à m'écrire. ${ }^{1}$

Mais en octobre, le livre sera déjà chez les libraires. Si $\mathrm{M}^{\text {gr }}$ de Solages avait pu imaginer que le contrat avec le Seuil était pratiquement signé, il en aurait été effrayé ${ }^{2}$. Son conseil porte sur le Phénomène humain, le livre qui avait été façonné à Carmaux. Son allure scientifique pourrait constituer un atout. Dans cet ouvrage, Teilhard accorde à l'homme une place centrale: dans le monde, l'Homme est entré sans bruit $[. . .]^{3}$. Loin d'être une réédition de l'humanisme d'autrefois, Teilhard ne décrit pas la création du premier homme mais son entrée dans le monde. Cet homme n'est pas statique, il est axe et flèche de l'évolution ${ }^{4}$. Cette pensée prend appui sur cette vision évolutive, ce qu'il faut absolument mettre en avant au moment de publier le livre. Dans des courriers successifs, $\mathrm{M}^{\mathrm{gr}}$ de Solages s'inquiète et demande avec insistance si l'avertissement se trouve bien en tête de l'ouvrage. C'est pour lui un élément capital mais pas suffisant. Il faut prendre davantage de précautions si l'on veut que cette publication échappe aux sanctions romaines. Les deux cercles que nous avions vus se dessiner du vivant de Teilhard existent toujours, non sans quelques

\footnotetext{
${ }^{1}$ Lettre de Br. de Solages à J. Mortier, 24 avril 1955 in: FTdC.

${ }^{2}$ Les éditions du Seuil attendaient depuis le début des années 1950 le feu vert pour publier les œuvres de Teilhard de Chardin. Paul Flamand et Teilhard étaient proches, notamment depuis que le Seuil avait publié le livre d'une des sœurs de Teilhard, M.-M. Teilhard de Chardin, L'Énergie spirituelle de la souffrance. Le jésuite s'était ainsi lié avec Paul Flamand, qu'il définissait comme un éditeur de livres chrétiens "intelligents", dans sa lettre à N. M. Wildiers, le 29 novembre 1952 in: KADOC.

${ }^{3}$ P. Teilhard de Chardin, Le Phénomène Humain, p. 22.

${ }^{4}$ P. Teilhard de Chardin, Le Phénomène Humain, p. 11.
} 
frictions. Jeanne Mortier d'une part et le tandem Solages - Lubac d'autre part, n'ont pas la même perception des éventuelles sanctions romaines qui pourraient frapper cette édition posthume. Ils parviennent malgré tout à s'entendre dans les grandes lignes.

Jeanne Mortier prend aussi une autre décision importante au cours de cette première réunion du mois d'avril 1955. Elle souhaite étoffer ce comité institué par Teilhard en y faisant entrer, dans un premier temps, quelques pères jésuites. Le P. Leroy sera le premier à y entrer, suivi du P. Russo ${ }^{1}$. De nouveau, elle se tourne vers $\mathrm{M}^{\text {gr }}$ Solages et le consulte sur ce point. $\mathrm{M}^{\text {gr }}$ de Solages y verrait plutôt des scientifiques. Ces deux jésuites sont un bon compromis mais il faudra, par la suite, rendre une place accrue aux hommes de science dans ce comité. Comme il a l'habitude de le faire, Bruno de Solages tient au courant son ami Henri de Lubac:

Je trouve votre lettre au retour de 3 jours d'absence (j'ai prêché à Aiguebelle une retraite spirituelle à un groupe de députés M.R.P.). Je trouve encore une longue lettre de $M^{\text {lle }}$ Mortier qui me promet les textes demandés, me demande si je crois pouvoir donner mon nom au Comité d'honneur de la publication du Phénomène humain. Je lui réponds de n'y mettre aucun ecclésiastique, aucun homme politique, mais seulement des savants ("toute l'Académie des sciences si vous pouvez"). ${ }^{2}$

Il semblerait que Jeanne Mortier soit en train de constituer un comité d'honneur qui viendrait apporter son soutien à la publication. Dans sa quête effrénée, elle n'a pas su s'arrêter. La liste s'allonge et, au milieu des clercs et des hommes politiques, la présence des scientifiques tend à se diluer. $\mathrm{M}^{\mathrm{gr}} \mathrm{de}$ Solages était venu l'alerter sur ce point. Elle se décide alors à établir deux listes, celles qui se trouvent désormais en tête des œuvres de Teilhard publiées aux éditions du Seuil. Ce qui dans un premier temps devait constituer un Comité d'honneur sera finalement scindé en deux, le Comité scientifique demeurant réservé aux savants, et le Comité général pour les autres personnalités. Dans les faits, uniquement le petit comité s'occupe de la publication ${ }^{3}$. Les savants qui figurent dans ces listes ont été contactés et leur signature a été sollicitée précisant qu'il s'agit d'un hommage, un hommage à l'homme et au penseur qui n'entraine pas nécessairement une adhésion aux formes et aux thèses de la

\footnotetext{
${ }^{1}$ Pierre Leroy, né en 1900, était entré dans la Compagnie de Jésus en 1920. Il suit des cours des sciences à l'Université de Nancy, auprès de Lucien Cuénot. En 1930, il est envoyé en Chine où il retrouve Teilhard. Les deux jésuites avaient fait connaissance à Paris, à la fin des années 1920. P. Leroy, Pierre Teilhard de Chardin tel que je l'ai connu, revient sur cette rencontre et sur ses liens avec Teilhard. François Russo était entré dans la Compagnie en 1933. Ancien élève de l'École Polytechnique, il en devient l'aumônier entre 1945 et 1950 avant d'être nommé rédacteur aux Études. Une notice sur lui est consultable in: G.-H. Baudry, Dictionnaire des correspondants de Teilhard de Chardin, pp. 108-109.

${ }^{2}$ Lettre de Br. de Solages à H. de Lubac, 5 juin 1955 in: CAECHL.

${ }^{3}$ Ce petit comité défini par Teilhard dans le testament a été élargi en 1955, après sa mort, à six personnes, dont voici les noms: Jeanne Mortier, Jean Piveteau, André George, Claude Cuénot, François Russo et Pierre Leroy.
} 
pensée elle-même ${ }^{1}$. Les premiers noms proviennent du carnet d'adresses de l'abbé Breuil ${ }^{2}$. Ces scientifiques, à leur tour, suggèrent d'autres noms. Le paléontologue Alberto Carlo Blanc, par exemple, répond ainsi à la demande qui lui est adressée:

Je vous remercie pour votre lettre $d u 29$ août, contenant la liste des personnes qui composent le Comité Scientifique. Je voudrais appeler votre attention sur le fait que cette liste ne comprend pas un des meilleurs amis et collaborateurs du Père Teilhard: le Prof. George B. Barbour, Dean of the College of Art and Science, University of Cincinnati, Ohio, USA. Le Prof. G. H. R. von Koenigswald, qui est mon hôte à Rome, pense comme moi que le prof. Barbour devrait être invité à en faire partie.

Une autre personnalité qu'il serait également opportun d'inviter à faire partie du Comité est le Dr. Paul Fejos, Directeur of Research of the Wenner Gren Foundation for Anthropological Research, 14 East $71^{\text {st }}$ St., New York, 21, N.Y., USA. C'est bien grâce à lui, en effet, que la Wenner Gren Foundation a largement aidé le P. Teilhard pendant ses dernières années. ${ }^{3}$

Nous voyons comment le réseau tend à s'internationaliser. Chaque retard dans l'édition, annoncé par le Seuil, est l'occasion d'ajouter de nouveaux noms à cette liste. Claude Cuénot, devenu le bras droit de Jeanne Mortier, s'adresse aux scientifiques en ces termes:

Nous avons l'intention de publier très prochainement un des principaux ouvrages du R. P. Teilhard de Chardin, le Phénomène humain, et désirerions inscrire en première page une double liste:

1) Un comité scientifique comprenant les principaux savants compétents en la matière, ceux qui étaient particulièrement estimés du R. P. Teilhard de Chardin.

2) Un comité d'honneur de l'Association des Amis du

R. P. Teilhard de Chardin.

Accepteriez-vous de voir paraitre votre nom dans la deuxième liste, et dans ce cas, auriez-vous la bonté de m'indiquer les titres que nous pourrons faire figurer à la suite de votre nom? ${ }^{4}$

La plupart répondent favorablement, mais pas tous. Victor Carlhian, professeur

\footnotetext{
${ }^{1}$ Lettre de C. Cuénot à V. Carlhian, 21 juin 1955 in: ÉF.

${ }^{2}$ De nombreuses références se trouvent dans les lettres échangées entre l'abbé Breuil et Jeanne Mortier au mois d'avril 1955 in: FTdC.

${ }^{3}$ Lettre d'A. C. Blanc à J. Mortier, 9 septembre 1955 in: FTdC.

${ }^{4}$ Lettre de C. Cuénot à V. Carlhian, 21 juin 1955 in: ÉF.
} 
de mathématiques à Lyon, adresse une réponse au conditionnel: $C$ 'est très volontiers que je verrai mon nom figurer dans le Comité d'honneur, à la condition toutefois que vous puissiez obtenir le nihil obstat et l'imprimatur, ce qui malgré le climat actuel me paraît possible. La parfaite rectitude des membres catholiques du Comité vis-à-vis de l'autorité religieuse serait ainsi sauve. ${ }^{1}$

Carlhian exige que l'œuvre ait reçu l'imprimatur, l'aval des autorités ecclésiastiques, avant d'accepter que son nom figure sur cette liste. Avec cette demande, il touche un point sensible. Tout religieux doit demander cette autorisation d'imprimer. De plus, depuis le début du XX $\mathrm{XX}^{\mathrm{e}}$ siècle, cette demande d'imprimatur doit être précédée d'un nihil obstat, mesure de sécurité supplémentaire ajoutée par Pie X en pleine crise moderniste. L'accord de deux ecclésiastiques ayant autorité dans l'Église est donc nécessaire pour que la publication soit autorisée ${ }^{2}$. Cet imprimatur sera demandé mais, sera-t-il accordé?

\subsection{En quête d'imprimatur}

L'obtention de l'imprimatur faisait partie du programme de Jeanne Mortier. Elle s'était placée dans la suite de Teilhard et voulait, en tout point, se montrer soumise à l'autorité de l'Église. L'édition de l'CEuvre était confiée aux éditions du Seuil. Comme il s'agissait d'un éditeur parisien, l'imprimatur devrait être sollicité auprès de l'ordinaire du lieu, en l'occurrence le cardinal Feltin. Jeanne Mortier se rapproche de l'archevêché de Paris et entame les premières démarches en vue de l'obtention de cette autorisation mais elle essuie un refus ${ }^{3}$. $\mathrm{M}^{\mathrm{gr}}$ de Solages est indigné. Dès qu'il connaît cette décision, il rédige un long courrier à l'attention du cardinal - courrier inutile, car la décision était prise -, mais nous lisons dans ces lignes les arguments adoptés par les amis de Teilhard, défenseurs de ce que Teilhard nommait la cause:

De toutes manières on peut craindre la mise à l'Index, ce qui sera un effroyable scandale (au sens théologique du terme) car ici, pour le monde entier, le P. Teilhard apparait comme porte-drapeau de la Science. Le danger d'interdire peut être pire si le livre parait sans imprimatur car ce livre ne contient absolument rien contre la foi et les mours.

Je me porte garant et je ne puis que confirmer ce que j'ai dit à Mlle Mortier que je suis prêt à signer le Nihil obstat. Il y a un quart de siècle que je me bats

\footnotetext{
${ }^{1}$ Lettre de V. Carlhian à C. Cuénot, 29 juin 1955 in: ÉF.

${ }^{2}$ Cf. D. Le Tourneau, Imprimatur in: Ph. Levillain (éd.), Dictionnaire historique de la papauté, p. 854.

${ }^{3}$ Cf. Lettre de J. Diot à J. Mortier, 20 juin 1955 in: FTdC. Jeanne Mortier note l'issue de l'entretien qui lui a été accordé par le cardinal Feltin au dos de cette lettre. C'est la seule trace écrite qui demeure de cette demande d'imprimatur. Les méandres de cette quête sont expliqués in: M. Prats, Le Teilhardisme, ch. 1 \& 4, et envisagés en parallèle avec la recherche d'imprimatur qui a occupé ces cercles de teilhardiens dans la suite de l'édition de l'œuvre, notamment pour le Milieu divin, en 1957.
} 
pour cette cause. Ce n'est pas le moment de me défiler! ${ }^{1}$

$\mathrm{M}^{\mathrm{gr}}$ de Solages se dit prêt à apposer son sceau sur cette publication et pour cela, il dispose d'un atout: la renommée de Teilhard. Au lendemain du décès, les journaux n'ont cessé de retracer sa carrière, la Croisière Jaune, la découverte du Sinanthrope, l'entrée à l'Académie des Sciences, etc. Bon nombre de librespenseurs attendent l'Église au tournant. La menace d'une nouvelle affaire Galilée circule ${ }^{2}$. Une condamnation de son livre serait perçue comme un refus par l'Église de reconnaître les idées évolutionnistes. La justesse de cette affirmation importe moins que le fait que l'opinion publique y soit attachée et Rome est sensible à cet argument. $\mathrm{M}^{\mathrm{gr}}$ de Solages le rappelle au cardinal Feltin dans sa lettre: Je sais que cet argument porte. Je l'ai déjà employé il y a 7 ans dans une lettre à Ottaviani et le voyant quelques mois après, il m'a dit soyez tranquille pour vos savants, nous ne renouvelons pas l'affaire Galilée. ${ }^{3}$

Au début du mois de juillet 1955, Jeanne Mortier signe un contrat avec les éditions du Seuil. Ce contrat stipule que seront publiés, dans l'année, le Phénomène humain et le Milieu divin ainsi qu'une série d'essais mineurs, rassemblés dans un volume. Elle n'a pas obtenu l'accord de l'Église pour publier mais la liste de noms qu'elle a réussi à associer à cette publication lui donne des ailes. Le 8 juillet 1955, Jeanne Mortier annonce à l'éditeur comment elle entend se passer de l'imprimatur:

Á défaut d'imprimatur pour le $\mathrm{Ph}$. $\mathrm{H}$., nous aurons un Comité scientifique imposant: le Président de l'Académie des Sciences, de grands noms de l'Institut et du Collège de France, les de Broglie, les premiers paléontologistes du monde entier. Une censure du S. Office équivaudrait à une condamnation de la Science. Vous recevrez aussi, très prochainement, cette liste, et celle du Comité d'Honneur. Toutes deux devront figurer en tête $d u$ Phénomène Humain. ${ }^{4}$

Le Phénomène humain reçoit l'imprimatur de la science à défaut de celui de l'Église. Y figurent une vingtaine de scientifiques français comme l'abbé Breuil, professeur honoraire de Préhistoire au Collège de France, Louis de Broglie ainsi que son frère Maurice de Broglie, tous deux membres de l'Académie des sciences, Louis Fage, président de l'Académie des Sciences et Robert Courrier, secrétaire perpétuel de ladite Académie, Louis Leprince-Ringuet, professeur à l'École polytechnique, Paul Rivet, Raymond Vauffrey, etc. À tous ces grands noms français viennent s'ajouter d'autres comme le professeur Von Koenigswald, de l'Université d'Utrecht ou Arnold Toynbee, professeur à Londres ainsi que quelques noms venus des États-Unis comme le professeur

\footnotetext{
${ }^{1}$ Lettre de Br. de Solages au cardinal Feltin, le 15 juin 1955 in: Archives privées de la famille de Solages.

${ }^{2}$ Les œuvres de Copernic et celles de Galilée sont restées à l'Index jusqu'en 1835. Cf. A. Fantoli, Problèmes historiques posés par la clôture de la question galiléenne en 1992, p. 109.

${ }^{3}$ Lettre de Br. de Solages au cardinal Feltin, 15 juin 1955 in: Archives privées de la famille de Solages.

${ }^{4}$ Lettre de J. Mortier aux éditions du Seuil, 8 juillet 1955 in: IMEC.
} 
George Barbour, professeur de géologie à Cincinnati, ou le professeur George Gaylord Simpson, de l'Université de Columbia. Ils n'étaient pourtant pas des inconditionnels de Teilhard. Mais à l'occasion de cette édition posthume, ils acceptent tous volontiers d'apposer leur nom, de rendre ce dernier hommage.

À côté de ce comité scientifique, le comité d'honneur - devenu finalement comité général - sera soutenu par de grandes personnalités, majoritairement des professeurs de philosophie, Gaston Bachelard, Étienne Borne, Gaston Berger, Jean Hyppolite, Jean Lacroix ou Jean Wahl et des hommes de lettres comme Robert Aron, Georges Duhamel. Certains sont aussi connus pour leur engagement politique comme André Malraux, Léopold Sédar Senghor, Roland de Margerie ou Jacques Rueff. Certains d'entre eux sont connus pour être catholiques, par exemple Étienne Borne, mais pas tous. D'autres noms viendront s'ajouter au tome 2 et suivants, comme par exemple celui du paléontologue catalan Miquel Crusafont i Pairó; d'autres noms disparaîtront pour cause de décès, comme l'abbé Breuil ou Gaston Berger; d'autres encore souhaiteront se retirer une fois passé le temps des hommages - nous y reviendrons.

\subsection{Le bouclier scientifique}

Pour le moment, cette caution apportée par ces scientifiques constitue un puissant bouclier. Jeanne Mortier et tous ceux qui portent cette publication, attendent les réactions venues de Rome mais leur attente est loin d'être passive. Le Phénomène humain est sur le point de paraître. Pour $\mathrm{M}^{\mathrm{gr}}$ de Solages il est capital qu'il parvienne au pape en avant-première, avec un mot qui vienne lui rappeler l'importance de cette publication, notamment dans les milieux scientifiques. Il faut éviter à tout prix que la presse s'empare de la nouvelle avant que Pie XII n'ait pu se faire son opinion. Mais cette fois, la coordination entre les deux cercles de continuateurs de vocation fera défaut. Tout peut encore arriver.

Pie XII mène son pontificat avec fermeté. Il ne nomme pas de secrétaire remplaçant après le décès de $\mathrm{M}^{\mathrm{gr}}$ Maglione, voulant gérer lui-même toutes les questions ${ }^{1}$. Il était important de s'adresser au pape directement, surtout sachant que son Encyclique Humani generis passait pour avoir visé Teilhard de Chardin tout particulièrement ${ }^{2}$. Solages agit à distance. Il ne sait pas que les mois d'été ont ralenti les travaux d'édition et que l'exemplaire relié blanc, celui qui sera spécialement conçu pour être offert au pape, n'est toujours pas terminé à la fin du mois d'octobre. Jeanne Mortier lui cache ces retards à répétition.

Pendant ce temps, il faut trouver la personne idoine pour aller présenter l'ouvrage au pontife. Le paléontologue C. A. Blanc est le premier pressenti. Il est très touché par cette proposition qu'il préfère cependant décliner, ayant publié dernièrement quelques articles polémiques dans la presse italienne. Il conseille alors d'autres noms: l'abbé Breuil ou le prof. Leprince-Ringuet sont bien plus qualifiés que moi pour accomplir cette tâche délicate ${ }^{3}$, répond-il.

\footnotetext{
${ }^{1}$ Cf. A. Ricardi, Pie XII in: Ph. Levillain (éd.), Dictionnaire historique de la papauté, p. 1362.

${ }^{2}$ Cf. É. Fouilloux, Une Église en quête de liberté, p. 294.

${ }^{3}$ Lettre d'A. C. Blanc à J. Mortier, 9 septembre 1955 in: FTdC.
} 
Parmi les noms envisagés, les scientifiques français sont privilégiés: Lamarre, Viret, les frères Broglie ... . Aucune de ces pistes ne sera suivie. Ce sera finalement René Fontenelle, un ami de Solages qui venait d'être nommé évêque, qui sera chargé d'offrir l'exemplaire du Phénomène humain, fraichement édité, au pape $^{1}$. Cet envoi devrait être doublé d'une lettre d'accompagnement: Je pense que le comité scientifique devrait offrir le volume au St Père avec une lettre marquant l'importance qu'il attache à cet ouvrage qui donne de l'univers une vision conforme à la science et ouverte à la Révélation divine (sans faire la moindre allusion à la question d'imprimatur). ${ }^{2}$

Quand $\mathrm{M}^{\mathrm{gr}}$ de Solages écrit que le comité scientifique devrait préparer une lettre, il est en réalité convaincu qu'il est la personne la mieux indiquée pour le faire. L'affaire est bien trop importante pour qu'elle soit confiée à des laïcs, et encore moins à une femme. Ses échanges avec Henri de Lubac sont émaillés de remarques de ce type au sujet de Jeanne Mortier: Si on pouvait trouver quelqu'un qui lui fasse entendre raison. Je vais écrire à Cuénot, plus accessible à un raisonnement, ne serait-ce parce qu'il est un homme. ${ }^{3} \mathrm{M}^{\text {gr }}$ de Solages connait ces milieux romains et ne les imagine pas recevant des injonctions de la part d'une laïque sans mandat. Ce sont des spécialistes dans leur domaine qui ne sont pas prêts à déléguer le pouvoir ${ }^{4}$. Il fallait que ce discours soit spécialement conçu pour les toucher. Inquiet, il se met immédiatement à la tâche. Jeanne Mortier reçoit le brouillon au début du mois d'août ${ }^{5}$. Voici le début de la lettre:

Préoccupés, comme beaucoup de leurs collègues, de

l'harmonie à remettre sans cesse en évidence entre la

vision scientifique de l'univers qui se modifie toujours

et les vérités immuables de la Révélation, ils sont

heureux d'offrir à Votre Sainteté un exemplaire d'un

ouvrage capital qui va paraître: Le Phénomène

Humain, ouvrage écrit par un de leurs collègues que

Dieu vient de rappeler à lui et pour lequel ils gardent

la plus fidèle affection et la plus vive admiration: le R.

P. Teilhard de Chardin. ${ }^{6}$

Une fois l'ouvrage introduit, il est rapidement associé aux vues évolutionnistes: [...] nulle présentation de l'univers - du moins parmi celles qui tiennent compte de l'état actuel de la science - ne met plus à l'aise leur foi chrétienne, et il leur semble que cet ouvrage est destiné à faire une impression puissante et profondément favorable au Christianisme parmi les milieux scientifiques, poursuit $\mathrm{M}^{\mathrm{gr}}$ de Solages ${ }^{7}$. C'est en cela que le pape est sollicité, pour qu'il vienne

\footnotetext{
${ }^{1}$ Cf. Lettre de $\mathrm{M}^{\mathrm{gr}}$ Fontenelle à Br. de Solages, 21 octobre 1955 in: Archives privées de la famille de Solages.

${ }^{2}$ Lettre de Br. de Solages à J. Mortier, 8 juillet 1955 in: FTdC.

${ }^{3}$ Lettre de Br. de Solages à H. de Lubac, 29 novembre 1955 in: CAECHL.

${ }^{4} \mathrm{Cf}$. É. Fouilloux, Une Église en quête de liberté, pp. 248 sq.

${ }^{5}$ Cf. Lettre de Br. de Solages à J. Mortier, 5 août 1955 in: FTdC.

${ }^{6}$ Lettre de Br. de Solages à J. Mortier, 5 août 1955 in: FTdC.

${ }^{7}$ Cf. Lettre de Br. de Solages à J. Mortier, 5 août 1955 in: FTdC.
} 
apporter un regard bienveillant sur cette œuvre laquelle, faute d'être théologiquement impeccable, constitue une puissante apologétique.

Le pape ne lira jamais cette lettre. À la fin du mois d'octobre, Solages se rend à Paris et découvre que Jeanne Mortier avait rédigé sa propre lettre ${ }^{1}$. Devant cet événement imprévu, il a considéré qu'il fallait agir au plus vite. Le professeur Louis Leprince-Ringuet, physicien, a été sollicité et envoyé d'urgence à Rome. Ce savant rédigera alors son propre texte, en dehors de cet univers clérical, mais puisqu'il est trop tard pour s'adresser au pape, LeprinceRinguet écrit à un des membres du Saint-Office, le père Philippe de la Trinité: Je tiens d'abord à bien préciser que je n'ai aucune autorité pour émettre un avis sur son contenu doctrinal [Le Phénomène Humain]; n'ayant pas lu le manuscrit il ne m'est même pas possible de savoir dans quelle mesure des problèmes sont soulevés: de toute façon mon incompétence est très grande sur le plan théologique.

En revanche je pense connaître assez bien le milieu des hommes de science français, vivant parmi eux depuis plus de 25 ans, en contact constant avec l'Académie des Sciences, la Sorbonne, le Collège de France et les autres institutions homologues. ${ }^{2}$

Ce savant refuse de se battre sur le terrain théologique. Le temps du scientisme à la manière du $\mathrm{XIX}^{\mathrm{e}}$ siècle lui paraît révolu. Dans un contexte qu'il présente comme apaisé, Leprince-Ringuet explique qu'il ne faudrait pas qu'une sanction vienne ternir le regard que les scientifiques portent sur la foi, regard qu'il voit sur la voie de l'amélioration depuis quelques années, notamment grâce à l'influence de Teilhard. Il poursuit:

Le Père Teilhard comptait beaucoup d'amis dans cette bande marginale (des incroyants ou des convertis récemment) en particulier. Il suscitait l'intérêt et l'affection par sa foi d'enfant alliée à sa valeur de scientifique et à cette éclatante droiture qui lui conférait un rayonnement particulier. Nous l'avions élu à l'Académie des Sciences à une large majorité, confirmant cette sympathie. ${ }^{3}$

Il est intéressant de lire que Teilhard était entré à l'Académie des Sciences en 1950 grâce à ses travaux scientifiques, qui ont une réelle importance, mais aussi parce qu'il suscitait l'affection parmi ses confrères. C'est ici que le teilhardisme trouve ses racines: l'homme est indissociable de son œuvre. C'était ce que ses supérieurs avaient compris lorsqu'ils l'avaient encouragé à se présenter à Rome en 1948. En cas de condamnation de l'œuvre, ce rayonnement

${ }^{1}$ L'affolement de $M^{\text {gr }}$ de Solages laisse supposer que cette lettre a été envoyée mais nous ne pouvons pas l'affirmer tant que les archives de Pie XII demeurent fermées à la recherche.

${ }^{2}$ Lettre de L. Leprince-Ringuet à P. de la Trinité, 9 octobre 1955 in: ASJF.

${ }^{3}$ Lettre de L. Leprince-Ringuet à P. de la Trinité, 9 octobre 1955 in: ASJF. 
de la personne, resté dans les mémoires des scientifiques, conduirait non pas une nouvelle affaire Galilée mais - ce qui serait bien pire -, à une affaire Teilhard:

Je souhaite très vivement, mon Père, qu'une interdiction n'intervienne pas, même si elle pouvait trouver justification, car elle risquerait de susciter une "affaire Teilhard" au sein des milieux scientifiques et de provoquer bien des dégâts - hostilité ou amertume - qui seraient difficilement réparables avant longtemps. ${ }^{1}$

Quelle gloire tirerait l'Église de la condamnation d'un homme tout juste décédé, amplement apprécié et proposant une œuvre qui montre le chemin vers la foi? L'initiative de Jeanne Mortier avait plongé $\mathrm{M}^{\mathrm{gr}}$ de Solages dans une terrible angoisse. Il décide alors de soutenir l'intervention de Leprince-Ringuet avec l'envoi d'une série de lettres: au P. de Gorostarzu, le bras droit du Père Général de la Compagnie de Jésus à Rome, au cardinal Ottaviani et au P. Paul Philippe, membres du Saint-Office, à $\mathrm{M}^{\text {gr }}$ Montini ${ }^{2}$. Chaque lettre est personnalisée, elle porte un discours adapté à l'interlocuteur. C'est une incroyable course contre la montre qu'il mène, de manière à avertir ces prélats avant que la presse ne s'en charge. Pourtant, l'opinion publique sera un précieux allié de cette publication.

\section{Une contestation différée}

Très peu de voix osent s'élever contre cette publication menée à titre posthume ${ }^{3}$. Une certaine pudeur vis-à-vis de l'homme décédé retient quelques plumes. Son histoire est connue, il avait été interdit de publication. Comment oser s'en prendre à lui maintenant qu'il n'est plus là pour s'expliquer? La réaction romaine s'était produite en amont et avait été somme toute mineure ${ }^{4}$. Elle se limitait à déconseiller l'acquisition de l'ouvrage. Cette décision, parvenue aux oreilles de $\mathrm{M}^{\mathrm{gr}}$ de Solages, est perçue comme une victoire: $J^{\prime} a i$ un peu l'impression que nous allons éviter l'Index. Rome aurait demandé aux évêques de ne pas l'introduire dans les séminaires. Ce qui n'aurait pas de sens si on devait le mettre à l'Index. Gardez tout cela discrètement. ${ }^{5}$

Au mois de décembre, un article dans la Civiltà Cattolica regrettait que ces écrits n'aient pas été ensevelis avec son auteur, décédé au mois d'avril ${ }^{6}$. Il en faudrait bien plus pour arrêter Jeanne Mortier dans son élan. En France, la presse s'empare de la nouvelle de cette publication. L'œuvre, connue jusqu'ici

\footnotetext{
${ }^{1}$ Lettre de L. Leprince-Ringuet à P. de la Trinité, 9 octobre 1955 in: ASJF.

${ }^{2}$ Ces lettres sont datées de fin octobre-début novembre 1955 in: Archives privées de la famille de Solages.

${ }^{3}$ Un argus de la presse a été consulté et il n’y a pas de critiques publiques envers Teilhard de Chardin ni envers son œuvre avant l'année 1956.

${ }^{4}$ Cf. Lettre du Cardinal Ottaviani à $\mathrm{M}^{\mathrm{gr}}$ Liénart, le 8 octobre 1955 in: AHAP: [La Suprême Congrégation] attire spécialement l'attention de Votre Éminence sur le danger qu'il y aurait à laisser entrer ce livre dans les Séminaires et les Collèges catholiques et à le mettre dans les mains des étudiants de nos Facultés.

${ }^{5}$ Lettre de Br. de Solages à H. de Lubac, le 13 novembre 1955 in: CAECHL.

${ }^{6}$ Cf. G. Bosio, Il fenomeno umano nell'ipotesi dell'evoluzione integrale.
} 
uniquement par ceux qui faisaient partie du cercle de lecteurs de polycopiés, est désormais accessible. L'homme et l'œuvre sont indissociables dans ce devenir posthume: Il a fallu la mort de son auteur, le jour de Pâques de cette année, pour que commence, avec ce premier volume, la divulgation d'une æuvre dont on connaissait l'importance sans qu'il fût permis d'en avoir aisément communication. ${ }^{1}$

Le Figaro, Combat, le Monde, tous les journaux publient leur compte rendu de lecture et ne tarissent pas d'éloges. La mort de Teilhard le jour de Pâques apparait comme un clin d'œil de la providence, voire comme un signe évident de sa sainteté. Cependant, certains s'interrogent déjà sur la nature de l'ouvrage. Ce n'est pas parce que Teilhard écrit dans son avertissement qu'il faut lire ce livre comme un mémoire scientifique qu'il faudrait le croire. Le physicien Georges Ambrosino, chroniqueur à ses heures dans le Monde, émet des doutes:

Si le projet de l'auteur fut de composer une somme scientifique - ce qu'il affirme en plusieurs endroits, demandant qu'on lise son livre non comme un traité de métaphysique, ni un essai théologique, mais comme un mémoire scientifique, - alors il a échoué, et non pour des raisons de détail, comme par exemple l'emploi relâché du mot énergie, mais pour des raisons qui tiennent aux principes mêmes de la science. ${ }^{2}$

L'auteur de ces lignes est conquis par ce livre et se demande pourquoi fallait-il que son contenu ait besoin d'être soutenu par la science. Teilhard aurait commis une erreur de méthode, envisager le christianisme en tant que phénomène biologique 3 . Quelque réticence empêche Ambrosino de suivre Teilhard jusqu'au bout de son exposé, sans toutefois y être hostile. Voici ses mots de fin: Acceptons-le comme un mythe où le cour d'un homme a caché, sous les traits du mystère, toute son expérience, son inquiétude, son inébranlable foi ${ }^{4}$ Cette foi, empreinte d'une idée de progrès à la manière de celle du XIX ${ }^{\mathrm{e}}$ siècle, se drape d'idées évolutionnistes. Pour Ambrosino, il s'agit de l'ouvrage d'un savant qui devient apologète en cours de route. Nous le voyons, aucune critique sévère envers cet ouvrage ne transparaît dans la presse, même si des doutes sont émis sur sa nature scientifique.

\subsection{Une timide remise en cause}

Cette presse, grande alliée des premiers moments, servira aussi de relais à toutes sortes de commentaires sur la postérité de cette œuvre. Les différents volumes sont publiés, les uns après les autres. Teilhard de Chardin est l'auteur qu'il faut avoir lu. Des passages du Phénomène humain peuvent être cités de manière approximative ou donnant des références peu précises. Des citations

\footnotetext{
${ }^{1}$ A. Rousseaux, Le Phénomène humain in: FTdC.

${ }^{2} \mathrm{G}$. Ambrosino, Une théorie chrétienne de l'évolution in: FTdC.

${ }^{3} \mathrm{G}$. Ambrosino, Une théorie chrétienne de l'évolution in: FTdC.

${ }^{4} \mathrm{G}$. Ambrosino, Une théorie chrétienne de l'évolution in: FTdC.
} 
apparaissent parfois dans des lieux inattendus. Voici, à titre d'exemple, ce que publie la Vie Parisienne en 1958:

Un romancier catholique sortait récemment d'un théatre de striptease. Le rencontrant comme il mettait le pied sur le trottoir, un de ses amis jugea l'occasion excellente de le taquiner un peu et lui reprocha de se complaire à un spectacle qu'il ê̂t dî fuir.

- Vous croyez? dit l'écrivain. Lisez donc ceci.

En tirant de son portefeuille un morceau de papier, il mit sous les yeux de son interlocuteur le petit texte que voici:

Voir! On pourrait dire que toute la vie est là, sinon finalement, du moins essentiellement. Voir, c'est être plus. La perfection d'un animal, la suprématie d'un être ne se mesurent-elles pas à la pénétration et au pouvoir synthétique de leur regard? Chercher à voir plus et mieux n'est donc pas une fantaisie, un luxe, une curiosité. Voir ou périr!

- Ces lignes, ajouta-t-il, sont du R. P. Teilhard de Chardin. Une autorité qui $m$ 'autorise, semble-t-il, à chercher à voir "plus et mieux" ... Même quand il $s$ 'agit de ces jolies filles que je tiens pour la meilleure preuve de l'existence de Dieu! ${ }^{1}$

En quelques années, Teilhard est devenu l'auteur à la mode, celui qu'il faut citer, qu'on l'ait lu ou pas. Malgré la légèreté des propos, l'auteur de ces lignes a bien saisi le but poursuivi par le jésuite-paléontologue: apporter la meilleure preuve de l'existence de Dieu, note- $\mathrm{t}-\mathrm{il}^{2}$. Les écrits de Teilhard de Chardin sont utilisés à tort et à travers. L'illustre jésuite est une icône. C'est ici que des timides réactions de la part des scientifiques commencent à poindre. La nature scientifique du Phénomène humain est fort contestable et Jean Rostand semble être le seul à s'insurger publiquement. Dans un article du Figaro littéraire, il soutenait qu'il serait temps de faire table rase de ces contes de fées pour grandes personnes ${ }^{3}$. Il ne refuse pas l'évolution comme hypothèse mais affirme ne pas détenir la clé des origines ni l'explication de l'avenir de l'humanité. À la lecture de cet article, André Frossard, converti à la foi catholique et devenu son plus ardent défenseur, en profite pour affirmer que les scientifiques rejettent maintenant les théories de l'évolution ${ }^{4}$. Son style d'écriture est assorti à celui des dessins qu'il produit. Son sens de l'humour apporte toujours une note de fraîcheur à ces débats: Adieu donc, Coelacanthe, poisson plein d'imagination, et qui, en trente mille siècles de persévérants efforts, avait réussi à accéder à la

\footnotetext{
${ }^{1}$ [sans auteur], Une caution in: FTdC.

2 [sans auteur], Une caution in: FTdC.

${ }^{3} \mathrm{~J}$. Rostand, Répétons-nous, sur l'évolution, des contes de fées pour grandes personnes? in: FTdC.

${ }^{4}$ Cf. F. Gugelot, André Frossard.
} 
condition humaine, comme au bout d'une vie édifiante, le petit mousse décroche la casquette de capitaine au long cours! ${ }^{1}$

Ces débats sur les théories de l'évolution dépassent le cadre scientifique pour devenir un argument pour ou contre la foi catholique et semblent quitter, du moins provisoirement, le domaine de la science. La publication de l'Euvre se poursuit et Jeanne Mortier s'attaque désormais à l'édition du Milieu divin, le livre de piété de Teilhard de Chardin, autre versant de son œuvre. Pendant ce temps, le Phénomène humain est publié en anglais, dont l'introduction a été confiée à Julian Huxley, ami de Teilhard, mais surtout important théoricien de l'évolution ${ }^{2}$. S'il accepte de présenter l'ouvrage, nous pouvons constater, dans la préface, qu'il n'est pas prêt à suivre le jésuite jusqu'au bout:

Bien que de nombreux scientifiques puissent se
trouver, comme moi, dans l'impossibilité de le suivre
jusqu'au bout dans sa tentative courageuse de
réconcilier les éléments surnaturels du christianisme
avec les faits et les implications de l'évolution, cela ne
lui retire pas la valeur positive de son approche
naturaliste.

Le livre avait tardé à paraître en Grande Bretagne. Aucune des versions proposées par le traducteur ne semblait convenir à Jeanne Mortier, très soucieuse de retranscrire parfaitement la pensée de Teilhard. Mais ce retard est faible comparé aux incidents que cette édition rencontre en Espagne. Le paléontologue Miquel Crusafont i Pairó rejette la traduction qui en a été faite et, faisant preuve d'une redoutable ténacité, il parvient à en obtenir le retrait de la vente. El Fenómeno Humano ne paraît qu'en 1963. Il est intéressant de lire ce que Crusafont écrit à son sujet, dans l'introduction à la traduction espagnole, huit ans après l'édition française:

L'œuvre «scientifique» du P. Teilhard, malgré qu'elle
plonge ses racines dans la Paléontologie, a pénétré
tous les domaines avides et assoiffés de l'humanisme
contemporain. [...] Terminons cette partie de notre
introduction en affirmant que l'œuvre du père Teilhard
porte, avant tout, une intention spiritualiste et même
apologétique.

Ce sont les mots de conclusion d'une longue introduction dans laquelle le paléontologue dit toute son admiration pour l'homme et son œuvre mais, c'est surtout dans ce dernier paragraphe qu'il situe la nature de l'ouvrage: une

${ }^{1}$ A. Frossard, Preuves in: FTdC.

${ }^{2}$ Cf. J. Huxley, Evolution: the Modern Synthesis.

${ }^{3}$ J. Huxley, Introduction, p. 20: Though many scientists may, as I do, find it impossible to follow him all the way in his gallant attempt to reconcile the supernatural elements in Christianity with the facts and implications of evolution, this in no way detracts from the positive value of his naturalistic general approach.

${ }^{4}$ M. Crusafont i Pairó, Prólogo, p. 12: La obra "científica" del P. Teilhard, a pesar de partir de la Paleontología, ha penetrado todos los campos ávidos y sedientos del humanismo contemporáneo. [...] Terminemos esta parte de nuestra introducción afirmando que la obra del P. Teilhard lleva, ante todo, una intención espiritualista y aun apologética. 
apologétique. La ponctuation est tout aussi parlante car, lorsqu'il écrit æuvre «scientifique», il entoure ce mot de guillemets, attirant sur lui une attention particulière et le remettant en question ${ }^{1}$. Parallèlement, en France, la nature de l'œuvre scientifique de Teilhard, et celle du Phénomène humain en particulier, est à nouveau débattue. Quelques ouvrages s'y intéressent, ce qui est en soi un fait nouveau, après plusieurs années de consensus tacite.

Certes, un monitum avait été prononcé en 1962 par Jean XXIII, à la veille de l'ouverture du concile Vatican II, à l'encontre de l'œeuvre de Teilhard de Chardin et ses disciples mais l'importance de cet avertissement - telle est la traduction de monitum - a été excessivement mise en avant par une partie de la presse. En effet, intégristes et anticléricaux, pour des raisons radicalement opposées, ont monté en épingle cette mesure disciplinaire, les uns pour discréditer Teilhard, les autres pour pointer du doigt une Église qui ne connaîtrait rien à la science. Cette mise en garde n'a été qu'une victoire à la Pyrrhus pour les défenseurs de la tradition car elle a surtout contribué à attirer l'attention sur le cas de Teilhard ${ }^{2}$. La lecture de la presse donne cette impression; les chiffres des ventes du Phénomène humain en attestent. En 1955, 17.153 exemplaires ont été vendus. Le chiffre demeure stable durant quelques années avant de retomber à 7.000 ou 8.000 exemplaires par an à la fin des années 1950. Mais l'année 1962 les éditions du Seuil enregistrent 24.875 exemplaires vendus. Ce pic ne sera plus jamais dépassé ${ }^{3}$. Cette œuvre intrigue. Mais cet élargissement du lectorat amène aussi, inévitablement, la contestation.

\subsection{Teilhard, théoricien de 1'évolution ou faussaire?}

Quelques auteurs semblent se donner le mot et publient, au même moment, des ouvrages sur l'œuvre scientifique de Teilhard de Chardin: François Meyer, Jean Piveteau, Pierre Leroy, Louis Barjon ... . En réalité, c'est l'ensemble de la pensée de Teilhard qui est débattue. La revue Esprit, par exemple, lui consacre un important dossier en 1963 et, devant la réaction des lecteurs - et aussi des teilhardiens, mécontents de ne pas avoir été consultés - renouvelle son expérience en $1964^{4}$. Suivant le fil de notre enquête sur le Phénomène humain, nous portons plutôt notre regard sur cette discussion amorcée par les scientifiques, restés particulièrement discrets jusqu'ici, mais il était important de souligner que ce phénomène de remise en question de l'œuvre de Teilhard ne se limite pas à son versant scientifique.

\footnotetext{
${ }^{1}$ La traduction de l'avertissement par le professeur Crusafont trahit sa pensée. Certainement par accident, il introduit une négation dans la phrase qui inverse le sens de l'avertissement. Cf. M. Prats, El Fenómeno humano de Teilhard de Chardin ... .

${ }^{2}$ M. Prats, Le Teilhardisme, ch. 6, est entièrement consacré à l'explication du monitum, le commentaire du document, sa contextualisation et sa réception. De même, le terme intégriste y est défini suivant l'article d'É Fouilloux, Brève histoire de l'intégrisme au XX siècle. L'usage de ce terme est ici pertinent. Ce n'est qu'après Vatican II que les intéressés préfèrent être qualifiés de traditionnalistes, pour montrer qu'ils se réclament de la tradition de l'Église, celle qui remonte à Pie V.

${ }^{3}$ Chiffres des ventes des éditions du Seuil communiqués au P. Russo, le 19 décembre 1980 in: ASJF.

${ }^{4}$ Cf. Esprit, les numéros de mars 1963 et 1964.
} 
François Meyer avait cité rapidement Teilhard dans sa Problématique de l'évolution ${ }^{1}$. Dix ans plus tard, à la demande de l'Association des Amis de Teilhard en Belgique, il prépare un opuscule consacré exclusivement au cas de Teilhard, Les Grandes Dérives du monde vivant ${ }^{2}$. Pour Meyer, Teilhard avait le sens évolutionniste sans qu'on puisse le considérer comme théoricien de l'évolution. En tout cas, Teilhard aborde des problèmes qui dépassent les limites de la pensée scientifique. Jean Piveteau, de son côté, serait moins catégorique. Dans un petit ouvrage de vulgarisation, le Père Teilhard de Chardin savant ${ }^{3}$, il vante les mérites de la synthèse teilhardienne, synthèse de l'homme de science et de l'homme de foi ${ }^{4}$. Piveteau consacre quelques pages à la biographie puis conduit son récit vers le versant scientifique de l'œuvre. Selon lui Teilhard s'intéresse à l'évolution comme méthode d'étude des êtres mais il n'est pas, encore une fois, un théoricien de l'évolution. Pour Piveteau, la distinction entre science et philosophie est le plus souvent un artifice purement scolaire, la vraie

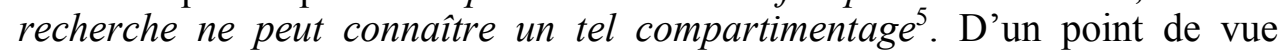
philosophique, il défend le Phénomène humain et rejette toute tentative de dissociation. L'œuvre de Teilhard est une puissante synthèse.

La même année, deux jésuites, Pierre Leroy et Louis Barjon, publient $L a$ carrière scientifique de Pierre Teilhard de Chardin et reviennent sur la nature du Phénomène humain. Cette publication a son importance quand on sait les réticences exprimées par la Compagnie de Jésus pour accepter cette publication sans imprimatur. Louis Barjon avait terminé d'écrire un ouvrage sur Teilhard le 10 avril 1956 - un an, jour pour jour, après le décès de son confrère jésuite mais ce livre ne franchit pas la censure interne à la Compagnie et se voit remisé. Le père Barjon ne renonce pas. Voyant que, en 1962, la Compagnie autorise le père de Lubac à publier son livre sur Teilhard ${ }^{6}$, Barjon rappelle que le sien est toujours inédit. Il obtient alors l'autorisation de publier son livre mais cette autorisation est partielle. En effet, l'opuscule qu'il signe avec Pierre Leroy en 1964 n'est qu'un tiré-à-part d'un premier volume censuré ${ }^{7}$. Ils n'ont publié que les pages qui concernaient le scientifique, dans lesquelles nous lisons ceci au sujet du Phénomène humain:

Résumé et synthèse, d'une part, de ses connaissances scientifiques; prolongement spirituel, par ailleurs, de ces mêmes connaissances et anticipation prophétique sur le devenir de l'Homme. Un livre qui forme charnière entre l'œuvre proprement dite du savant et

\footnotetext{
${ }^{1}$ Cf. F. Meyer, Problématique de l'Évolution.

${ }^{2}$ Cf. F. Meyer, Les Grandes Dérives du monde vivant.

${ }^{3}$ Cf. J. Piveteau, Le Père Teilhard de Chardin savant.

${ }^{4}$ J. Piveteau, Le Père Teilhard de Chardin savant, p. 3.

${ }^{5}$ J. Piveteau, Le Père Teilhard de Chardin savant, p. 103.

${ }^{6}$ Cf. H. de Lubac, La Pensée religieuse du P. Teilhard de Chardin.

${ }^{7}$ Cf. L. Barjon, Le Combat de Pierre Teilhard de Chardin.
} 
les essais de réflexion religieuse dont Teilhard entreprendra parallèlement la rédaction. ${ }^{1}$

La définition n'est pas clairement établie. Il s'agirait d'une forme charnière. Ces différents ouvrages tentent d'apporter des réponses mais il y a un manque évident de transparence. À force de tourner autour de la question et de se demander si le Phénomène humain est, oui ou non, un mémoire scientifique, c'est toute l'œuvre, celle du géologue et du paléontologue, qui est remise en cause, alors qu'elle existe et est conséquente ${ }^{2}$. Toutes ces palinodies, qui durent depuis 1955, avaient fini par agacer aussi les défenseurs de la foi catholique. En 1962, le groupe intégriste Action-Fatima publie un dossier touffu, Teilhard de Chardin contre la science, et tranche de manière radicale. La carrière du jésuite-paléontologue repose sur deux découvertes majeures: l'Éoanthropus ou Homme de Piltdown et le Sinanthrope ou Homme de Pékin. Pour ActionFatima, elles sont toutes deux facilement contestables:

Pour le premier, l'homme de Piltdown, il a bien un crâne, mais la mâchoire qui lui a été mise pour en faire un être à caractères simiens est une mâchoire de chimpanzé vieille de 50 ans qu'on a teintée pour qu'elle ait l'air fossile. Teilhard y a ajouté lui-même une dent limée et teintée pour les besoins de la cause.

Pour le deuxième, l'homme de Pékin, il se base sur des reproductions modelées ou sculptées dans lesquelles la fantaisie et l'esprit de parti ont joué plus que la vérité. ${ }^{3}$

Nous ne reviendrons pas sur l'affaire de Piltdown, indéniablement une fraude qui a marqué l'histoire de la science ${ }^{4}$. Pour la presse intégriste, l'occasion est trop belle pour venir déconstruire ce personnage mythique qui a pris forme au fil des années. À leurs yeux, il n'y a que deux possibilités. Dans le premier cas, Teilhard de Chardin avait volontairement fraudé, ce qui est peu louable. Dans le deuxième cas, il s'était laissé abuser, ce qui ne met pas en avant ses qualités de scientifique. Les livrets d'Action-Fatima sont distribués à la sortie des églises pour informer les catholiques qui se laisseraient entraîner par le teilhardisme.

\footnotetext{
${ }^{1}$ Cf. L. Barjon \& P. Leroy, La Carrière scientifique de Pierre Teilhard de Chardin, p. 105.

${ }^{2}$ Teilhard avait dressé lui-même sa liste de Titres et travaux en vue de son admission au Collège de France. Cette liste de mémoires scientifiques est consultable à la Fondation Teilhard de Chardin. Elle a été publiée, par la suite, dans le tome 13 de l'Euvre, par les éditions du Seuil, mais cette liste a été manipulée par Jeanne Mortier, laquelle a, par exemple, exigé de l'éditeur qu'il retire les références à l'Homme de Piltdown alors que, en 1948, Teilhard en faisait état puisque la fraude n'avait pas été constatée que quelques années plus tard.

${ }^{3}$ [sans auteur], Teilhard de Chardin contre ..., p. 80 in: FTdC. Action-Fatima se constitue en communauté autour de l'abbé Jean Boyer, en 1960. En 1962, ils mènent une campagne antiteilhardienne. Le cardinal Feltin leur interdit toute nouvelle publication en mai 1962. Cf. P. Airiau, Jean Boyer. Cette interdiction prononcée par le cardinal Feltin, en mai 1962, conduirait à penser que cette brochure a été éditée dans la première moitié de l'année 1962, juste avant le monitum.

${ }^{4}$ Cf. M. Russell, The Piltdown Man Hoax: case closed, R. Ladous, Darwin, Teilhard, Sherlock Holmes et les autres: l'énigme de Piltdown in: ASJF \& H. Thomas, Le Mystère de l'homme de Piltdown.
} 
Ce pamphlet s'efforce de montrer la supériorité de la foi catholique sur les inepties teilhardiennes et sur la science en général. La pensée de Teilhard est présentée comme un système clos, un isme qui mériterait son nom, le teilhardisme. Louis Jugnet, professeur de philosophie à Toulouse, avait lancé le terme dans la presse au début de l'année $1956^{1}$, juste après la publication du Phénomène humain, mais qui y avait prêté attention? Pour le P. Philippe de la Trinité, membre du Saint-Office, teilhardisme rime avec progressisme, l'un se nourrissant de l'autre ${ }^{2}$. De tous les côtés, le teilhardisme est discuté, ce qui, dans l'opinion publique, le rend d'autant plus attirant. La réception de cette pensée semble parvenir à un apogée au moment de la proclamation du monitum et bénéficie d'un sursaut en 1965, au moment où les teilhardiens sont heureux de commémorer les dix ans de la mort de Teilhard. Mais qui dit apogée annonce déjà le déclin.

\section{Le seuil de l'année 1965}

Le succès de cette vie posthume repose sur deux piliers: le paléontologue et le jésuite, la science et la foi. Ces deux piliers sont soutenus par le souvenir qu'avait laissé cet homme charismatique. Porter atteinte à l'un de ces éléments déconstruit l'ensemble. Après avoir vu la position scientifique de Teilhard discutée, Jeanne Mortier s'efforce de préparer un dixième anniversaire de la disparition de Teilhard qui vienne surpasser tous les autres. Elle l'avait fêté tous les ans mais, en 1965, quelques temps forts vont marquer l'année. La création à la Maison de la Radio de l'œuvre d'André Jolivet le Cœeur de la Matière, inspirée par une œuvre de Teilhard, est un succès ${ }^{3}$. La session qui se tient tous les ans à Vézelay depuis 1960 ne démérite pas. En revanche, tout ce qui touche au Teilhard scientifique semble compromis.

Maurice Vernet publie, en 1965, La Grande illusion de Teilhard de Chardin. Il ne voit que des erreurs dans cette œuvre et exige qu'elle soit dépouillée de tout aspect mythique, une véritable légende s'est établie autour de l'cuvre et de la personne de Teilhard ${ }^{4}$, note-t-il avant de s'appliquer à déconstruire le personnage. Pourtant, Paul Chauchard soutient le contraire et verrait même chez Teilhard un saint Thomas du $\mathrm{XX}^{\mathrm{e}}$ siècle ${ }^{5}$. Arrivés à ce stade, il n'est plus possible de citer toutes les publications qui s'intéressent à cet incroyable phénomène de société qu'est le teilhardisme. L'automne de l'année 1965 semble être le moment choisi par les contradicteurs pour discuter la pensée scientifique de Teilhard. Nous en retiendrons trois moments saillants.

\subsection{La science dixit}

La revue Itinéraires lance, en 1965, une grande enquête au sujet de

\footnotetext{
${ }^{1}$ Cf. L. Jugnet, Le système du monde du P. Teilhard de Chardin in: FTdC.

${ }^{2}$ Cf. P. de la Trinité, Rome et Teilhard de Chardin, p. 210.

${ }^{3}$ Cf. M. Prats, Le Teilhardisme, ch. 8.

${ }^{4}$ M. Vernet, La Grande illusion de Teilhard de Chardin, p. 12.

${ }^{5}$ Cf. P. Chauchard, La Foi su savant chrétien, par exemple, mais aussi de nombreux articles dans la presse. Il défend une lecture thomiste de l'œuvre de Teilhard.
} 
Teilhard. Ce n'est pas la première fois que ce jésuite attire l'attention de cette revue mais c'est le premier dossier monographique ${ }^{1}$. Itinéraires consacre un premier numéro à Teilhard et la religion, au mois de mars, avant d'aborder Teilhard et la science dans son numéro de septembre-octobre. Jean Madiran avait fondé cette revue une dizaine d'années auparavant. Avant d'étudier le contenu du dossier, il faut rappeler que cette revue se situe dans la mouvance intégriste. Ces défenseurs de l'Algérie française ne s'y retrouvent plus. Selon eux, le panorama politique est désastreux. Ils attendaient au moins une reprise en main de la part de l'Église à l'issue du concile Vatican II. Au lieu de cela, le teilhardisme fait école. Jean Madiran a considéré qu'il était temps de dresser une liste de questions et de les poser aux personnes compétentes. Il adresse alors un questionnaire aux scientifiques les plus en vue et les interroge sur l'œuvre scientifique de Teilhard.

Il faut d'abord souligner que le fait d'aborder la pensée de Teilhard en deux numéros est déjà une forme de déconstruction de l'image posthume de Teilhard, laquelle se désagrège irrémédiablement quand on ne tient pas compte de son ensemble, y compris la bonhomie du jésuite-paléontologue. Enfin, s'intéresser à l'œuvre scientifique de Teilhard revient à incriminer aussi la science. La science dixit $^{2}$, écrit Madiran, pour exprimer sa colère devant cette manière de l'utiliser comme caution et comme justification à tout, y compris de nouvelles théologies. Voici le questionnaire:

L'enquête de la revue ITINERAIRES demande aux hommes de science dans quelle mesure ils peuvent répondre, chacun du strict point de vue de sa discipline scientifique, aux questions suivantes:

1. Y a-t-il un rapport proprement scientifique de Teilhard de Chardin dans la discipline envisagée, et en quoi consiste-t-il?

2. Monogénisme et polygénisme:

a) Ya-t-il accord unanime, ou divergence, du point de vue de la discipline scientifique envisagée, sur le fait que l'humanité descend d'un premier homme ou bien de plusieurs rameaux préhumains ayant abouti à une pluralité de races?

b) Faut-il parler à ce sujet de certitude scientifique ou d'hypothèse scientifique?

3. Y a-t-il continuité ou non-continuité de la matière à l'esprit? La continuité, ou la non-continuité, est-elle

\footnotetext{
${ }^{1}$ L. Salleron avait publié plusieurs articles au sujet de l'œuvre de Teilhard dans Itinéraires: une critique du Phénomène humain en mars 1956, une autre à propos du Groupe zoologique humain en mai 1956, sur le Milieu divin dans le numéro de septembre-octobre 1958, un commentaire sur la Pensée religieuse du P. Teilhard, le livre du P. de Lubac, en juin 1962 et un commentaire aussi sur le livre de Jean Onimus, Teilhard ou la foi au monde en mai 1963. Par ailleurs, le P. Calmel publie La distinction des trois ordres en mars 1962, il critique Construire la terre de Teilhard en avril 1962, Réponse au teilhardisme en décembre 1963, Lumière du dogme et brume du teilhardisme en décembre 1963. Cette liste est rappelée in: Itinéraires, septembre-octobre 1965.

${ }^{2}$ Cf. [sans auteur], Teilhard et la science in: FTdC.
} 
une hypothèse scientifique ou une certitude scientifique?

4. L'évolutionnisme: quel est le sens de ce mot dans la discipline envisagée? Quelle est la part de l'hypothèse et celle de la certitude? Y a-t-il plusieurs systèmes évolutionnistes s'excluant les uns les autres, ou peuton au contraire concevoir, par-delà les divergences, un évolutionnisme commun aux divers systèmes scientifiques? ${ }^{1}$

Nous n'avons pas eu accès aux archives de cette revue. Nous ne savons pas quels sont les scientifiques qui ont été contactés et lesquels auraient éventuellement refusé de répondre ${ }^{2}$. Nous n'avons que les réponses publiées, dont certaines expriment déjà un refus à leur manière ${ }^{3}$. Deux d'entre eux, par exemple, adressent des articles qu'ils ont déjà publiés ailleurs, ce qui revient à ne pas répondre au questionnaire ${ }^{4}$. Ces deux dissidents sont Jean Piveteau et Jean Rostand ${ }^{5}$. Nous comprenons mieux la position de Rostand quand on sait qu'il préparait, au même moment, une intervention dans Le Figaro littéraire; nous la retrouvons dans le paragraphe suivant.

Le professeur Simpson, professeur à l'Université de Harvard, répond de manière rapide et particulièrement efficace:

Si par la première question, vous voulez dire: "le Père Teilhard de Chardin, a-t-il ajouté à la connaissance des faits relativement à l'étude des fossiles?", la réponse est "oui". Si vous voulez dire: "A-t-il contribué, d'une manière absolument scientifique, à expliquer l'évolution ou la théorie de l'évolution?", la réponse est "non". En fait, il a confondu les deux sujets. $^{6}$

Ce sera tout. Pour Simpson, la question 2 est sans importance scientifique et aussi encore une fois mal posée, la question 3 est complètement dépourvue de substance scientifique tout comme la question 4. Est-ce la revue qui l'agace? Est-ce Teilhard? Ou les deux? Rappelons que Simpson avait accepté, dans un

\footnotetext{
${ }^{1}$ [sans auteur], Teilhard et la science in: FTdC.

${ }^{2}$ Présent, journal proche d'Itinéraires aurait été une piste à suivre mais notre demande de consultation de leurs archives, en date du 15 mai 2019, est restée, pour le moment, sans réponse.

${ }^{3}$ Voici la liste des réponses, dans l'ordre de publication dans Itinéraires: Louis Bounoure, professeur de biologie à l'Université de Strasbourg, Raymond Arthur Dart, Professeur à Witwatersrand, Michel Delsol, professeur de biologie à l'Université catholique de Lyon, Félix Manix, professeur à l'Université de Vienne et directeur de l'Institut de biologie générale de la Faculté de médecine, Pierre Niaussat, chef de la direction de Biologie générale et écologie du Centre de recherches du service de santé des Armées à Paris, André Reymond, directeur adjoint du Muséum d'Histoire naturelle de Paris, chef de laboratoire d'études au Muséum de Brunoy, Jean Rostand, biologiste et membre de l'Académie française, George G. Simpson, professeur au Museum of comparative Zoology à l'Université de Harvard, Jean Piveteau, professeur de Paléontologie à la Sorbonne.

${ }^{4}$ Une étude détaillée de toutes les réponses se trouve in: M. Prats, Le Teilhardisme, ch. 8.

${ }^{5}$ Ils ont tous deux adressé des articles datés de 1963, publiés dans la France Catholique pour Jean Piveteau et dans la Revue de Paris pour Jean Rostand.

${ }^{6}$ [sans auteur], Teilhard et la science in: FTdC.
} 
premier temps, de voir son nom figurer dans le Comité scientifique, celui qui présidait aux publications des éditions du Seuil. Cependant, depuis le tome 6, son nom n'y figure plus. Le temps des hommages est terminé. Avec lui, le professeur Reymond, du Muséum d'histoire naturelle de Paris, trouve aussi ces questions intempestives, insolubles et répond bien souvent par je ne sais pas car il est des certitudes qui ne sont pas scientifiques. Le professeur Niaussat, biologiste et chercheur au service de santé des Armées de Paris, donne une réponse laconique et le professeur Manix, biologiste à l'Université de Vienne, explique que ce sont surtout les ouvrages philosophico-théologiques de Teilhard qui ont eu du succès. Enfin, le professeur Dart, de l'Université de Witwatersrand, en Afrique du Sud, répond très clairement: la contribution de Teilhard de Chardin dans le Phénomène humain, est d'ordre philosophique. Ce dossier se réduit finalement à deux réponses conséquentes, celles de deux professeurs de biologie: Louis Bounoure, professeur à l'Université de Strasbourg, et Michel Delsol, professeur à l'Institut catholique de Lyon. Tous deux refusent aussi de répondre à ce questionnaire, ils répondent autrement.

Le rédacteur en chef de cette revue savait par avance que l'article de Bounoure ne viendrait pas défendre la pensée de Teilhard. Michel Delsol l'avait deviné puisqu'il y fait allusion dans son propre article:

Il existe en effet un spécialiste de la zoologie qui n'accepte pas la Théorie de l'Évolution. J'ai été parfois étonné, je l'avoue, en lisant les arguments que le Professeur Bounoure - auteur par ailleurs de travaux sur la sexualité et la reproduction qui font autorité, donne contre l'évolution biologique: je me permets de dire que l'on a l'impression que le Professeur Bounoure n'a pas suivi depuis quelques années la bibliographie paléontologique.

On ne peut pas soutenir par exemple, comme il l'a écrit dans plusieurs articles, que les documents paléontologiques que nous possédons donnent des images séparées les unes des autres. Nous avons au contraire sur des lignées entières des séries de squelettes qui se suivent de si près qu'il n'est plus possible, par exemple, de séparer autrement que de façon arbitraire les Poissons et les Batraciens, les Reptiles et les Mammifères. ${ }^{1}$

Nous comprenons, en lisant ces lignes, que le professeur Bounoure est effectivement un scientifique reconnu par ses pairs mais que, dans le cas particulier de l'évolution, il fait bande à part, il n'a jamais voulu adhérer à l'hypothèse transformiste dans aucune de ses variantes, darwinisme ou lamarckisme ${ }^{2}$. Après de longues pages, Bounoure revient à la conclusion de Simpson: il y aurait deux Teilhard. Le premier serait un bon technicien de

\footnotetext{
${ }^{1}$ M. Delsol, L'évolution biologique, p. 50 in: FTdC.

${ }^{2}$ Cf. L. Loison, French Roots of French Neo-Lamarckisms (1879-1985).
} 
l'histoire des primates tandis que le deuxième, porté par des vues imaginaires, aurait été un mauvais théoricien de l'évolution. Rappelons que pour Simpson, Teilhard n'était ni bon ni mauvais, il n'était tout simplement pas théoricien de l'évolution. Dans tous les cas, tout cela a été une source de confusions immenses et, dans cette confusion, l'avertissement du Phénomène humain ainsi que la manière dont l'édition posthume de l'œuvre a été conduite, y sont pour beaucoup. Jean Madiran laisse parler les scientifiques, sans ajouter d'autres commentaires. Le père Philippe de la Trinité saura lui apporter des lecteurs.

Le 17 septembre 1965, Jean Farran et Jean Namur consacrent une émission télévisée d'une heure à Pierre Teilhard de Chardin ${ }^{1}$. Le Teilhard scientifique est mis en avant, notamment avec les interventions de Pierre Leroy, Jean Piveteau et Louis Leprince-Ringuet. Mais lorsque le père de la Trinité est questionné, ce dominicain, membre du Saint-Office, n'a pas une seconde d'hésitation. Sans vouloir remettre en cause Teilhard, le teilhardisme lui paraît une doctrine inacceptable, cheval de Troie pour les marxistes. Il admet volontiers que le jésuite ait pu produire des conversions mais, au moment même où il dit que les catholiques feraient mieux de se former à meilleure école, la caméra montre des revues posées sur un muret. Le père de la Trinité les déplace délicatement. Il a amené avec lui toute la documentation nécessaire. Le dernier numéro d'Itinéraires se profile sur la partie droite de l'écran, posé sur sa page de couverture pour que le titre soit lisible sur la tranche. C'est indéniablement une subtile invitation à le lire, bien visible dans le film (un peu moins dans cette capture d'écran).

\subsection{Jean Rostand ouvre le feu}

Au même moment, Jean Rostand sème le trouble avec un article dans Le Figaro littéraire à la fin du mois de septembre. Le courrier des lecteurs a valu à cette revue un deuxième numéro pour essayer de calmer les émois. Nous ne reviendrons pas de manière détaillée sur cette publication que nous avons déjà commentée dans un article précédent ${ }^{2}$. Notons uniquement que Rostand n'y va pas par quatre chemins. Il annonce d'emblée qu'il n'existe pas de théorie teilhardienne de l'évolution ${ }^{3}$. L'idée d'évolution tient certes une grande place dans la pensée de Teilhard mais c'est tout. Rostand répond au courrier des lecteurs la semaine suivante. Retenons sa conclusion:

En bref, grand biologiste que Teilhard, grand explicateur de l'évolution? Non, mais profond moraliste et noble écrivain. Si l'on y ajoute les incontestables mérites du paléontologiste et du géologue, cela forme une personnalité d'une ampleur peu commune, et dont on n'aimerait à dire que du bien, et dont on ne dirait jamais que du bien si l'on n'avait parfois les oreilles

\footnotetext{
${ }^{1}$ Cf. J. Farran \& J. Namur, Pierre Teilhard de Chardin.

${ }^{2}$ Cf. M. Prats, 1965, l'apothéose de Teilhard de Chardin.

${ }^{3}$ Jean Rostand s'en prend à Teilhard de Chardin in: FTdC.
} 
fatiguées par les contresens des ignorants, les hyperboles des naïfs et les gloussements des snobs. ${ }^{1}$

Rostand rappelle quelles étaient les compétences scientifiques de Teilhard: paléontologie et géologie, mais refuse de le classer parmi les théoriciens de l'évolution. Il lui reconnaît aussi des qualités d'écrivain et un charisme hors du commun. Ce que Rostand regrette est, au fond, le teilhardisme, tout ce bruit que font les adeptes de cette doctrine et les commentateurs en tout genre et cette terrible confusion entre ce qui est scientifique et ce qui ne l'est pas dans cette œuvre. Le colloque qui se tient à l'UNESCO à la fin de l'année ne contribue pas à apaiser ce climat de contestation ${ }^{2}$.

\subsection{Teilhard et Einstein, un anniversaire commun}

Ce colloque devait commémorer la disparition, à seulement quelques jours d'intervalle, de ces deux savants, Albert Einstein et Pierre Teilhard de Chardin, et leur rendre hommage en parallèle. L'idée de convergence si chère à Teilhard retrouvait l'esprit de l'UNESCO et de son premier directeur, Julian Huxley. Puisque nous avons consacré un article à cet évènement ${ }^{3}$, nous dessinons ici uniquement comment ce qui avait été décidé dans un premier temps avec un enthousiasme certain prend, au fil des jours, mauvaise tournure. Les différents intervenants, ceux qui dans un premier temps avaient accepté de participer, écrivent. Des lettres demandant à prendre des distances avec Teilhard parviennent à l'UNESCO. Le secrétaire qui les reçoit fait part de son inquiétude à son supérieur hiérarchique: Beaucoup d'invités ont annoncé qu'ils refusaient de parler sous de telles auspices. Sir Julian Huxley lui-même a insisté pour que son nom ne soit pas lié à un hommage à Teilhard de Chardin. Sa conférence portera sur le thème de Science et synthèse et non sur l'œuvre de Teilhard. ${ }^{4}$

La réaction de Huxley n'est pas un cas isolé. Elle a conduit les organisateurs à modifier le programme et distinguer les journées. Le colloque a lieu les 13, 14 et 15 décembre. Les deux premiers jours seront réservés à Einstein pendant que les teilhardiens seront accueillis dans une plus petite salle le vendredi 15 décembre. Toute possibilité de synthèse entre ces deux penseurs est anéantie. Les amis de Teilhard mènent à terme leur journée. Jean Piveteau présente la synthèse teilhardienne et insiste sur la nouvelle dimension que Teilhard donne à l'humanisme, celle qui présente l'homme comme flèche de l'évolution. Le père Leroy plaide de son côté pour la convergence entre science et philosophie pendant que le professeur Chouard rappelle l'effet que produisaient les prises de parole de Teilhard parmi les étudiants dans les années 1920. Tous s'accordent sur le fait que son œuvre, Phénomène humain en tête, apporte l'explication que tant de scientifiques attendaient. Enfin, la philosophe Madeleine Barthélémy-Madaule situe cette pensée non pas dans l'entre-deux-

\footnotetext{
${ }^{1}$ Jean Rostand répond à ses contradicteurs in: FTdC.

${ }^{2}$ Dossier général Colloque Science et Synthèse in: UNESCO.

${ }^{3}$ Cf. M. Prats, La sintesis de Teilhard de Chardin ... .

${ }^{4}$ M. Bammate à M. Machado, 6 décembre 1965 in: UNESCO.
} 
guerres, ou du temps de son écriture, mais au moment de sa réception. Que peut le teilhardisme face au structuralisme? Elle dit, entre autres:

Je crois que c'est à cela que nous sommes conviés. Nous arrivons à une totalité qui n'est pas du tout la totalité que recherchent actuellement, par exemple, les structuralistes. On recherche une totalité dans la structure, une totalité dans l'analyse. Nous savons bien ce que c'est; c'est une totalité de type linguistique ou psychanalytique ou politique marxienne; chaque élément d'une structure renvoie à d'autres éléments de cette structure: c'est un renvoi perpétuel, jamais un sens ne se dégage! Or ce que cherche le Père Teilhard, c'est le "sens".

La synthèse de Teilhard apparaît pour ces lecteurs comme la preuve absolue que la vie a un sens. Devant le constat existentialiste, le teilhardisme apporte une réponse optimiste. Madeleine Madaule se bat pour rendre une place à cette vision mise à mal par la montée du structuralisme. Le paradigme évolutionniste est épuisé. Après Auschwitz et Hiroshima, l'idée d'un homme maître de son destin, glissant vers la perfection, n'est plus de mise. Le structuralisme inaugure une véritable révolution intellectuelle, notamment dans le domaine de l'anthropologie ${ }^{2}$. La publication des actes du colloque, en 1967, montre comment la place consacrée à Teilhard, par rapport à Einstein, s'était encore réduite. Les journées consacrées à Einstein sont détaillées dans la table des matières alors que celle consacrée à Teilhard occupe une toute petite place. Le teilhardisme s'essouffle. Il est en perte de vitesse, même si les amis de Teilhard s'efforcent de maintenir la flamme.

\section{Conclusion}

En 1965, Mircea Eliade donne une conférence à l'Université de Chicago et revient sur l'engouement suscité par le teilhardisme, auquel il associe le succès rencontré par Tristes Tropiques de Claude Lévi-Strauss. Eliade y voit une mode culturelle, une vogue qui serait née à Paris dans un premier temps, répandue rapidement dans toute l'Europe puis parvenue aux États-Unis. Fasciné par ce phénomène, il note qu'il n'importe guère que les faits et leur interprétation soient vrais ou non. Aucune critique ne peut venir à bout d'une vogue $e^{3}$. Ces deux penseurs, Teilhard de Chardin et Lévi-Strauss, réagissent contre l'existentialisme, tous deux exaltent la nature physique, une mythologie de la matière $^{4}$. Lévi-Strauss allait participer de ce moment structuraliste pendant que Teilhard, décédé, rendait possible la naissance du teilhardisme.

\footnotetext{
${ }^{1}$ M. Barthélémy-Madaule, Science et Synthèse, p. 367.

${ }^{2}$ Cf. F. Dosse, Histoire du structuralisme, p. 411.

${ }^{3}$ M. Eliade, Modes culturelles et histoire des religions, p. 13.

${ }^{4}$ M. Eliade, Modes culturelles et histoire des religions, p. 27.
} 
Au terme de cette enquête, la définition du teilhardisme se profile. Cet isme est un nuage diffus qui a traversé le cœur des Trente glorieuses ${ }^{1}$ et qui prend comme point de départ l'image mythique du jésuite paléontologue. Cette image est amplement relayée par la presse et par ses adeptes, les teilhardiens. Ils la rendent indissociable de l'œuvre, une œuvre à deux versants, scientifique et religieux. Ce sont bien ces trois facettes réunies - l'homme, le savant, le religieux - qui définissent le teilhardisme. Cet isme prospère tant qu'elles restent solidaires. Contester la nature scientifique du Phénomène humain n'est pas sans conséquences. L'ouvrage a été propulsé sur le devant de la scène à partir du moment où il a été décidé qu'il serait l'opus 1 de Teilhard. En absence d'imprimatur, il était trop risqué de s'aventurer dans la publication du Milieu divin, son œuvre de piété. Le Phénomène humain avait su toucher de nombreux esprits scientifiques. L'Église, et le Saint-Office en particulier, devraient être sensibles à cet argument. Ces signatures prestigieuses venues s'associer en signe de dernier hommage au moment de publier l'œuvre feront office d'imprimatur. Mais pour la légataire des écrits, c'était tout sauf un dernier hommage. La mort de l'homme avait ouvert l'œuvre à la vie.

La lecture de ce mémoire scientifique a été remise en cause somme toute assez tardivement. L'avertissement ajouté au Phénomène humain à l'issue du Concile de Carmaux est en grande partie responsable de cette confusion des genres. Teilhard de Chardin était un missionnaire jésuite. Ses illustres prédécesseurs apprenaient le chinois et partaient en mission dans des terres lointaines. Teilhard parlera le langage des scientifiques et s'efforcera de leur montrer le chemin qui mène à cette ultime convergence qu'il a décidé de nommer Oméga.

Mais finalement, dans cette aventure, il y a un perdant: son œuvre scientifique, celle qu'il a patiemment menée au fil de ses années de travaux de géologue et de paléontologue. Au moment où le Phénomène humain commence à être discuté, Teilhard est accusé de faussaire par ses contradicteurs. Le dossier Itinéraires a permis de fixer sur le papier l'avis fort tranché de quelques noms illustres. Le temps de l'hommage est passé, la menace d'une condamnation romaine s'efface car l'existence même de l'Index est en voie de disparition. L'œuvre de Teilhard n'a plus besoin de ce bouclier scientifique. Cette vague de teilhardisme qui avait déferlé sur la France et qui s'était étendue suivant la ligne de ces Trente glorieuses, s'estompe. Le teilhardisme avait ses limites. Le Phénomène humain, un mémoire scientifique? Certainement pas. Ce fut une puissante apologétique. Sartre était philosophe-écrivain, Teilhard était jésuitepaléontologue. Adroit et avers d'une même médaille, en cette période d'existentialismes, l'un prêche l'absurdité du monde, l'autre son aboutissement heureux. Le structuralisme s'est chargé de les départager.

\section{Bibliographie}

1. Sources

Barjon L., Le Combat de Pierre Teilhard de Chardin, Presses universitaires, Laval 1971.

\footnotetext{
${ }^{1}$ Cf. J. Fourastié, Les Trente glorieuses.
} 
Barjon L. \& Leroy P., La Carrière scientifique de Pierre Teilhard de Chardin, Éditions du Rocher, Monaco 1964.

Baudry G.-H., Dictionnaire des correspondants de Teilhard de Chardin, chez l'Auteur, Lille 1974.

Chauchard P., La Foi su savant chrétien, Aubier, Paris 1957.

Crusafont i Pairó M., Prólogo in: P. Teilhard de Chardin, El Fenómeno humano, transl. M. Crusafont i Pairó, Taurus, Madrid 1963, pp. 11-31.

Huxley J., Evolution: the Modern Synthesis, Allen \& Unwin, London 1942.

Huxley J., Introduction in: P. Teilhard de Chardin, The Phenomenon of Man, trad. B. Wall, Collins, London 1959, pp. 11-28.

Leroy P., Pierre Teilhard de Chardin tel que je l'ai connu, Plon, Paris 1958.

Lubac H. de, La Pensée religieuse du P. Teilhard de Chardin [1962], Cerf, Paris 2002.

Meyer F., Problématique de l'Évolution, PUF, Paris 1954.

Meyer F., Les Grandes Dérives du monde vivant, Éditions universitaires, Paris 1963.

Ouince R. d', Un prophète en procès: Teilhard de Chardin, Aubier, Paris 1970.

Piveteau J., Le Père Teilhard de Chardin savant, Fayard, Paris 1964.

Teilhard de Chardin M.-M., L'Énergie spirituelle de la souffrance, Seuil, Paris 1950.

Teilhard de Chardin P., Le Phénomène Humain, Seuil, Paris 1955.

Teilhard de Chardin P., Note sur quelques représentations historiques possibles du péché originel in: P. Teilhard de Chardin, Euvres complètes, vol. 10: Comment je crois, Seuil, Paris 1969, pp. 61-70.

Teilhard de Chardin P., Lettres à Jeanne Mortier, (éd.) S. Clair-Michot, Seuil, Paris 1984.

Trinité P. de la, Rome et Teilhard de Chardin, Arthème Fayard, Paris 1964.

Vernet M., La Grande illusion de Teilhard de Chardin, Gedalge, Paris 1965.

\section{Archives}

AHAP = Archives historiques de l'archevêché de Paris: 1D 15 29, documents $\mathrm{du}$ Saint-Office, R. P. Teilhard de Chardin, Le Phénomène humain, correspondance Ottaviani/Liénart.

Archives privées famille de Solages, Mézens: Correspondance de $\mathrm{Br}$. de Solages/cardinal Feltin, $\mathrm{M}^{\text {gr }}$ Fontenelle/Br. de Solages.

ASJF = Archives jésuites de la Province de France, Paris: Fonds Teilhard de Chardin 16, R. Ladous, Darwin, Teilhard, Sherlock Holmes et les autres: l'énigme de Piltdown, article de presse sans date.

ASJF $=$ Archives jésuites de la Province de France, Paris: Fonds Teilhard de Chardin 6-4.1, correspondance L. Leprince-Ringuet/Ph. de la Trinité; chiffres des ventes des éditions du Seuil communiqués au P. Russo.

CAECHL $=$ Centre d'Archives et d'Études cardinal Henri de Lubac, Namur: Correspondance de H. de Lubac/Br. de Solages.

ÉF = Archives privées Étienne Fouilloux: Correspondance: C. Cuénot/V. Carlhian.

FTdC $=$ Fondation Teilhard de Chardin, Paris: Correspondance de H. Breuil/J. Mortier, A. C. Blanc/J. Mortier, M. Diot/J. Mortier, Br. de Solages/J. 
Mortier, P. Teilhard de Chardin/R. d'Ouince, J. Mortier/J. Villain.

IMEC $=$ Institut Mémoires de l'édition contemporaine, Caen: Fonds Seuil SEL 1072: coupures de presse reçues par P. Flamand, avril 1955, la Croix, le Monde, Le Figaro et Combat: correspondance J. Mortier/éditions du Seuil.

$\mathrm{KADOC}=$ Katholieke Documentatie en Onderzoekscentrum, Louvain: Fonds N. M. Wildiers, section 15, correspondance P. Teilhard de Chardin/N. M. Wildiers.

UNESCO: Dossier général du colloque Science et Synthèse, du 13 au 15 décembre 1965: correspondance M. Bammate/M. Machado, M. Barthélémy-Madaule, Science et Synthèse, actes du colloque, Gallimard 1967.

\section{Littérature secondaire}

Airiau P., Jean Boyer in: J. P. Chantin (éd.), Les Marges du christianisme. Dictionnaire du monde religieux dans la France contemporaine, Beauchesne, Paris 2001, pp. 25-27.

Amadieu J.-B., La Littérature française au XIX siècle mise à l'Index. Les Procédures, Cerf, Paris 2017.

Barthélémy-Madaule M., Science et synthèse, Gallimard, Paris 1967.

Colin P., L'Audace et le Soupçon, Desclée de Brouwer, Paris 1997.

Dosse F., Histoire du structuralisme, La Découverte, Paris 1991.

Eliade M., Modes culturelles et histoire des religions [1967] in: M. Eliade, Occultisme, sorcellerie et modes culturelles, trad. J. Malaquais, Gallimard, Paris 1976, pp. 11-29.

Fantoli A., Problèmes historiques posés par la clôture de la question galiléenne en 1992 in: F. Beretta (éd.), Galilée en procès, Galilée réhabilité?, Éditions Saint-Augustin, Saint-Maurice 2005.

Fouilloux É., Une Église en quête de liberté, Desclée de Brouwer, Paris 1998.

Fouilloux É., Brève histoire de l'intégrisme au XX siècle in: É. Fouilloux, Les Chrétiens français entre guerre d'Algérie et mai 1968, Parole et Silence, Paris 2008, pp. 109-122.

Fourastié J., Les Trente glorieuses, Fayard, Paris, 1979.

Gugelot F., André Frossard in: L. Ducerf, V. Petit \& M. Tramaux (éd.), Dictionnaire du monde religieux de la France contemporaine. Franche-Comté, Beauchesne, Paris 2016, pp. 320-321.

Laplanche F., La Crise de l'origine. La Science catholique des Évangiles et l'histoire au XX' siècle, Albin Michel, Paris 2006.

Levillain Ph. (éd.), Dictionnaire historique de la papauté, Fayard, Paris 1994.

Loison L., French Roots of French Neo-Lamarckisms (1879-1985) in: Journal of the History of Biology vol. 44, 4/2011, pp. 713-744.

Poulat É., Histoire, Dogme et Critique dans la crise moderniste, Casterman, Paris 1962.

Prats M., 1965, l'apothéose de Teilhard de Chardin in: Religión, Laicidad y Sociedad en la historia contemporánea de España, Italia y Francia, (éd.) P. Álvarez Lázaro, A. Ciampani \& F. García Sanz, Universidad Pontificia de Comillas, Madrid 2017, pp. 183-200.

Prats M., El Fenómeno humano de Teilhard de Chardin, avatares de una publicación in: eVOLUCION, oct. 2017, pp. 65-72 [en ligne: http://sesbe.org/ 
wp-content/uploads/2016/01/eVOLUCION-122.pdf., consulté le 1 sept. 2019].

Prats M., La sintesis de Teilhard de Chardin, ¿una síntesis científica? El dilema del coloquio de la UNESCO en 1965, conférence donnée au VIII Coloquio internacional sobre Darwinismo en Europa y América. El evolucionismo en Canarias, Las Palmas de Gran Canaria, 15 al 18 de mayo 2018.

Prats M., Le Teilhardisme. Réception, Adoption et Travestissement de la pensée de Teilhard de Chardin à la croisée des sciences et de la foi au cour des «Trente glorieuses» en France (1955-1968), thèse dirigée par F. Gugelot [soutenance prévue pour le 7 décembre 2019 à l'université de Reims Champagne-Ardenne].

Russell M., The Piltdown Man Hoax: case closed, The History Press, Cheltenham 2012.

Thomas H., Le Mystère de l'homme de Piltdown. Une extraordinaire imposture scientifique, Belin, Paris 2002.

\section{Coupures de presse se trouvant à la Fondation Teilhard de Chardin,} Paris

Ambrosino G., Une théorie chrétienne de l'évolution. Le Phénomène humain in: le Monde, 13 décembre 1955.

Bosio G., Il fenomeno umano nell'ipotesi dell'evoluzione integrale (A proposito di un libro del p. Teilhard de Chardin) in: La Civiltà Cattolica, 17 décembre 1955, pp. 622-631.

Cuénot C., Pierre Teilhard de Chardin: Le Phénomène humain in: Combat, le 3 novembre 1955.

Delsol M., L'évolution biologique in: Itinéraires, septembre-octobre 1965.

Esprit, les numéros de mars 1963 et 1964.

Jean Rostand s'en prend à Teilhard de Chardin in: Le Figaro littéraire, 23-29 sept. 1965 , pp. $1 \& 10$.

Jean Rostand répond à ses contradicteurs in: Le Figaro littéraire, 12-20 oct. 1965.

Frossard A., Preuves, juin 1956.

Jugnet L., Le système du monde du P. Teilhard de Chardin in: La Nation française, 15 février 1956.

Rostand J., Répétons-nous, sur l'évolution, des contes de fées pour grandes personnes? Réflexions d'un biologiste autour de quelques ouvrages récents in: Le Figaro Littéraire, 20 avril 1957.

Rousseaux A., Le Phénomène humain in: Le Figaro littéraire, 24 décembre 1955.

[sans auteur], Teilhard de Chardin contre l'Évangile de Jésus-Christ, la Science in: Action-Fatima 1962.

[sans auteur], Teilhard et la science in: Itinéraires, septembre-octobre 1965.

[sans auteur], Une Caution in: la Vie Parisienne, juillet 1958.

\section{5. Émission télévisée}

Farran J. \& Namur J., Pierre Teilhard de Chardin, le 17 septembre 1965, 21:40, $1^{\mathrm{e}}$ chaine, 56 minutes CPF 86638989, INA. 